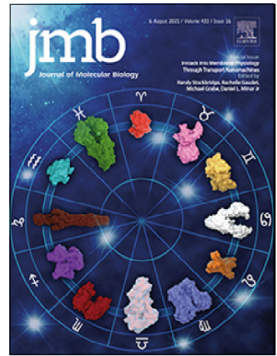

\title{
The Stability Landscape of de novo TIM Barrels Explored by a Modular Design Approach
}

\author{
Sergio Romero-Romero ${ }^{1,2}$, Miguel Costas ${ }^{3}$, Daniel-Adriano Silva Manzano ${ }^{4,5 \dagger}$, \\ Sina Kordes ${ }^{2}$, Erendira Rojas-Ortega ${ }^{1}$, Cinthya Tapia ${ }^{1}$, Yasel Guerra ${ }^{1}$, \\ Sooruban Shanmugaratnam ${ }^{2}$, Adela Rodríguez-Romero ${ }^{6}$, David Baker ${ }^{4,5 *}$, \\ Birte Höcker $^{2 *}$ and D. Alejandro Fernández-Velasco ${ }^{1 *}$
}

\author{
1 - Laboratorio de Fisicoquímica e Ingeniería de Proteínas, Departamento de Bioquímica, Facultad de Medicina, Universidad \\ Nacional Autónoma de México, 04510 Mexico City, Mexico \\ 2 - Department of Biochemistry, University of Bayreuth, 95447 Bayreuth, Germany \\ 3 - Laboratorio de Biofisicoquímica, Departamento de Fisicoquímica, Facultad de Química, Universidad Nacional Autónoma \\ de México, 04510 Mexico City, Mexico \\ 4 - Department of Biochemistry, University of Washington, 98195 Seattle, USA \\ 5 - Institute for Protein Design, University of Washington, 98195 Seattle, USA \\ 6 - Instituto de Química, Universidad Nacional Autónoma de México, 04510 Mexico City, Mexico
}

Correspondence to David Baker, Birte Höcker and D. Alejandro Fernández-Velasco: Institute for Protein Design, University of Washington, 98195 Seattle, USA (D. Baker), Department of Biochemistry, University of Bayreuth, 95447 Bayreuth, Germany (B. Höcker), Departamento de Bioquímica, Facultad de Medicina, Universidad Nacional Autónoma de México, 04510 Mexico City, Mexico (D. A. Fernández-Velasco).dabaker@uw.edu (D. Baker), birte.hoecker@uni-bayreuth.de (B.Höcker), fdaniel@unam.mx (D.A. Fernández-Velasco)

https://doi.org/10.1016/j.jmb.2021.167153

Edited by Amy Keating

\begin{abstract}
The ability to design stable proteins with custom-made functions is a major goal in biochemistry with practical relevance for our environment and society. Understanding and manipulating protein stability provide crucial information on the molecular determinants that modulate structure and stability, and expand the applications of de novo proteins. Since the $(\beta / a)_{8}$-barrel or TIM-barrel fold is one of the most common functional scaffolds, in this work we designed a collection of stable de novo TIM barrels (DeNovoTIMs), using a computational fixed-backbone and modular approach based on improved hydrophobic packing of sTIM11, the first validated de novo TIM barrel, and subjected them to a thorough folding analysis. DeNovoTIMs navigate a region of the stability landscape previously uncharted by natural TIM barrels, with variations spanning 60 degrees in melting temperature and $22 \mathrm{kcal}$ per mol in conformational stability throughout the designs. Significant non-additive or epistatic effects were observed when stabilizing mutations from different regions of the barrel were combined. The molecular basis of epistasis in DeNovoTIMs appears to be related to the extension of the hydrophobic cores. This study is an important step towards the fine-tuned modulation of protein stability by design.

(c) 2021 The Authors. Published by Elsevier Ltd. This is an open access article under the CC BY-NC-ND license (http://creativecommons.org/licenses/by-nc-nd/4.0/).
\end{abstract}




\section{Introduction}

Proteins are essential macromolecules capable of performing diverse and exquisite biological functions such as immune protection, cellular communication, or enzymatic reactions. To guarantee such activities, the functional states must act under specific environmental conditions in a relevant time scale, that is, proteins must be "stable". Protein stability is required to maintain functional structures and it enhances the ability of proteins to evolve new properties. ${ }^{1,2}$ The central role of proteins in the chemistry of life, as well as their increasing application in basic and applied research, implies that the understanding and manipulation of protein stability are highly relevant.

There are two main indicators of protein conformational stability at equilibrium. One is the difference of free energy between the native and unfolded states at a given temperature $(\Delta G)$, which is often obtained by chemical unfolding experiments carried out at $25{ }^{\circ} \mathrm{C}$. In addition, stability is also assessed in the context of thermal unfolding, where the unfolding temperature $\left(T_{m}\right)$, the temperature at the midpoint of the transition from native to the unfolded state, is the most common parameter employed to quantify stability. Both the $\Delta G$ and $T_{m}$ parameters, usually determined as criteria for a "stable" protein, are related with the enthalpy $(\Delta H)$ and heat capacity $\left(\Delta C_{P}\right)$ changes through the Gibbs-Helmholtz equation, which describes the variation of $\Delta G$ with temperature, the so-called "stability curve" of proteins. $^{3}$ Different mechanisms have been proposed to modify the stability curve of proteins, ${ }^{4}$ and numerous studies on natural proteins and their site-directed mutants have been used to rationalize the stability of thermophilic proteins and moreover to engineer thermostability. ${ }^{5}$

Historically, the design of stable proteins has been one of the main objectives of computational protein design. ${ }^{6}$ Several strategies, such as increasing the hydrophobic area in internal cores, improvement of water-protein interactions, the introduction of disulfide bridges as well as the addition of salt bridges, have been proposed. ${ }^{7-18}$ The design of de novo proteins can further enhance our understanding of the physicochemical properties that modulate stability. For example, although folding behavior has been only addressed for very few cases, the kinetic analysis of the folding mechanism of two de novo $\beta a$ proteins has revealed complex free energy surfaces. ${ }^{19,20}$ The fine-tuning of conformational stability, that is, the manipulation of the protein stability curve, is an open challenge for protein design and engineering. Such a goal requires a comprehensive characterization of de novo proteins, describing the combination of thermodynamic parameters that can be reached in a particular fold.

Within the different topologies that a protein can adopt, the TIM-barrel or $(\beta / a)_{8}$-barrel fold is one of the most abundant superfolds in nature. ${ }^{21}$ Based on proteomic analysis, the TIM-barrel domain is also close to the average size of proteins present in Escherichia coli. ${ }^{22}$ Besides, the TIM-barrel fold is one of the most successful topologies used in nature to host catalytic activities. Due to its large variety of functions and its ubiquity in different types of enzymes, the TIM barrel represents a suitable scaffold for protein design and engineering. ${ }^{23}$ For these reasons, its construction has been an important objective over the years. ${ }^{24-28}$ Recently, the successful design of a de novo four-fold symmetric TIM barrel was described: the sTIM11 protein. $^{29}$ Considering that the sTIM11 sequence is significantly different from the ones found in naturally occurring TIM barrels, the potential of this scaffold to explore new thermodynamic properties and functions is highly interesting. sTIM11 shows a high melting temperature $\left(T_{m}=80^{\circ} \mathrm{C}\right)$ but low conformational stability $\left(\Delta G_{25^{\circ} \mathrm{C}}=\sim 4 \mathrm{kcal} \mathrm{mol}^{-1}\right)$ when compared to natural TIM barrels. ${ }^{29-32}$ Since low conformational stability often results in high sensitivity to mutations and changes in the environment, this can limit the design of novel proteins with new functions. ${ }^{8}$ Thus, fine-tuning the stability of the sTIM11 scaffold is a prerequisite to functionalize and generate tailor-made barrels for applications in biochemistry, biotechnology, and medicine. In this work, a fixed-backbone design with a modular approach was used to generate a collection of de novo TIM barrels. Their thermodynamic and structural properties were characterized in detail, increasing our knowledge on how stability can be fine-tuned by design.

\section{Results and discussion}

\section{DeNovoTIM collection designed by modular repacking of a de novo TIM barrel}

The de novo protein STIM11 is an idealized fourfold symmetric TIM barrel of 184 residues, which was designed to include two cysteines that, however, did not form the intended disulfide bond (Figure 1). To avoid reactive free thiols, both residues were reverted to the residues in the original four-fold design (C8Q and C181V), resulting in sTIM11noCys. The base design DeNovoTIM0, which is the starting point for all further constructs in this work, additionally contains the changes W34V and A38G in all symmetry-related quarters. These residues are situated in every second $\alpha / \beta$-loop, and in STIM11, these tryptophan residues are the most highly solvent exposed. While different strategies have been explored to increase protein stability, ${ }^{8,18}$ here we focused on hydrophobic repacking. The structural analysis suggested three regions to be amenable to improvements in STIM11, one in the internal and two in the peripheral hydrophobic cores. The inner face of the circular sheet forms the internal core, whereas the outer face of the strands and 

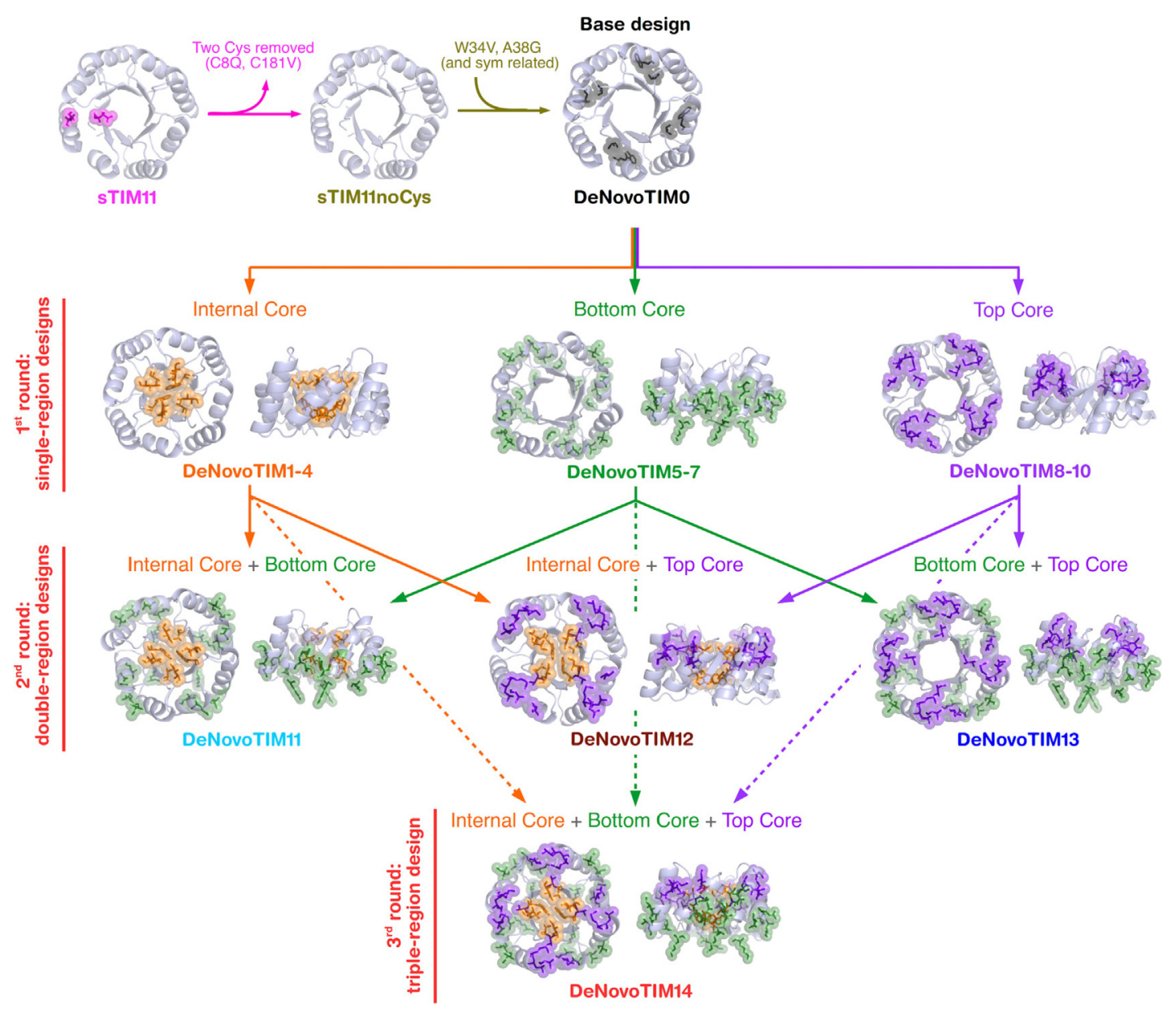

Figure 1. Modular design approach to obtain the DeNovoTIM collection. Cartoon representation of the regions and the corresponding residues modified in each design round. The two cysteine residues present in STIM11 that were reverted to the corresponding symmetry-related residues in STIM11noCys are shown in magenta (C8Q and C181V). Mutations W34V and A38G (as well as their 4-fold-symmetry related residues) introduced in DeNovoTIM0 are shown in black. The internal core, formed by the $\beta$-barrel residues A21, R23, 140, 142, A67, R69, I86, and I88 (as well as their 2 fold-symmetry related residues) is shown in orange. The bottom core, formed by the $\mathrm{N}$-terminal region of even $\beta$-strands and the C-region of the flanking a-helices, that is, residues Q11, E15, T18, K31, and V34 (as well as their 4-fold-symmetry related residues) is colored green. The top core situated at the C-terminal region of the odd $\beta$ strands and the N-terminal region of the flanking a-helices formed by residues K2, A5, W6, Y22, S24, and D29 (as well as their 4-fold-symmetry related residues) is shown in purple. All the sequences analyzed in this work are reported in Figure S2 and Tables S1 and S2.

the internal face of the helices constitute the peripheral core. In this latter, we identified two regions, henceforth named bottom and top cores as shown in Figure 1. The residues lining the three regions were subjected to fixed-backbone Rosetta design according to the flow diagram in Figure S1.

Ten designs were selected for characterization in the first round: four with modifications in the internal core (DeNovoTIM1-4) as well as three designs each for the bottom (DeNovoTIM5-7) and the top core (DeNovoTIM8-10) (Figure S2). For the internal core, no improved designs could be identified when four-fold symmetry was preserved. Therefore, in DeNovoTIM1-4 only a two-fold symmetry was enforced. An exploratory characterization by circular dichroism (CD) and differential scanning calorimetry (DSC) of DeNovoTIMs 0-10 showed that designs 1, 6, and 8 were the best for each region (Figure S3 and supporting text).

To test for additivity effects on stability and structure, mutations contained in the best design of each group were combined to generate the following double-region designs: DeNovoTIM11-13 as shown in Figure 1. Finally, in the third round the mutations of all three regions were combined resulting in DeNovoTIM14. All these proteins as well as sTIM11, sTIM11noCys, and DeNovoTIM0 were characterized in detail. Information on sequences, and mutations in each design are 

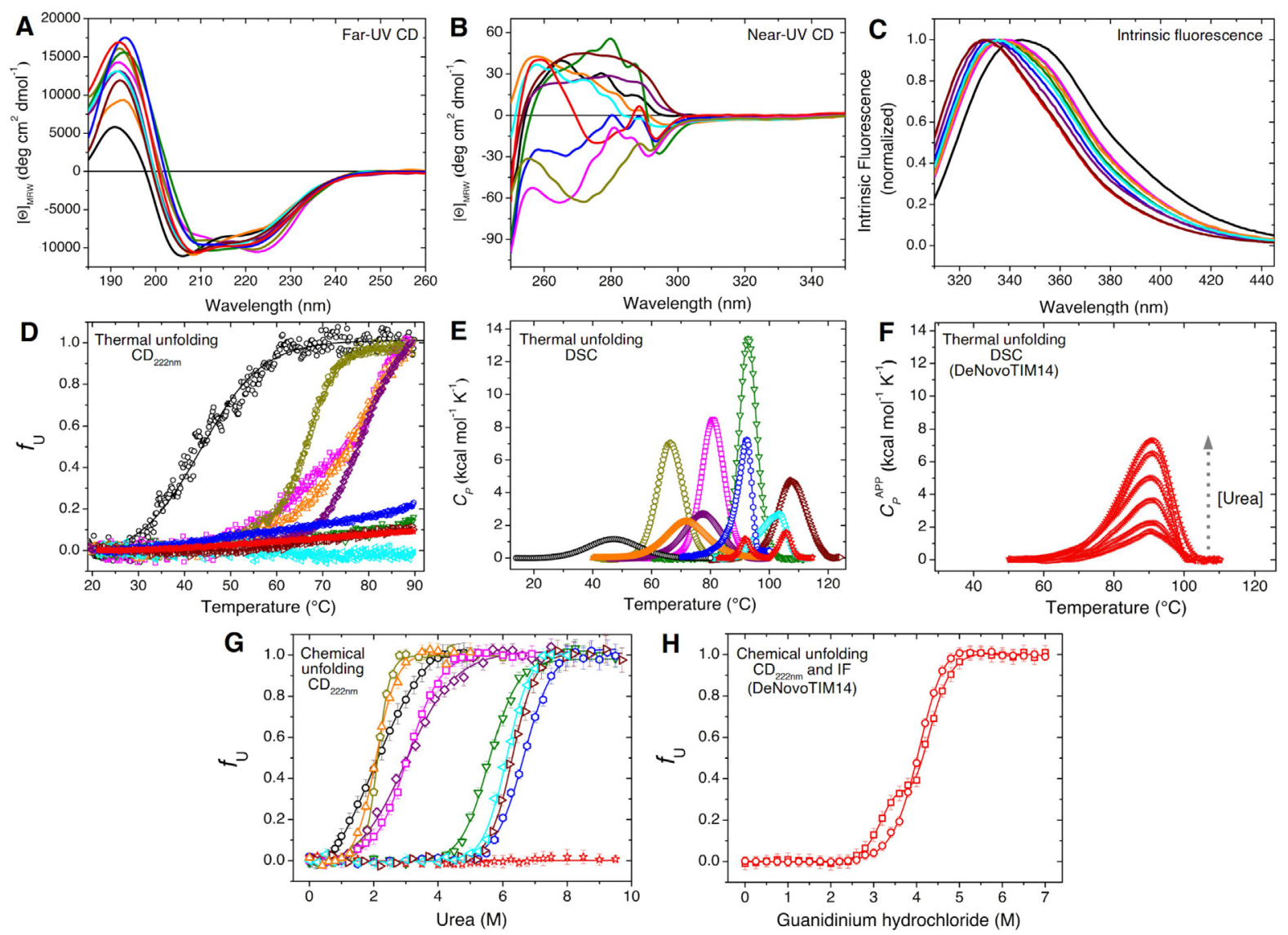

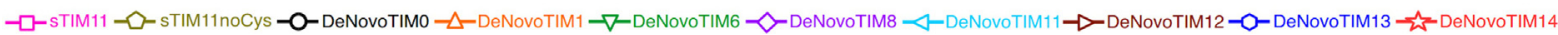

Figure 2. Conformational properties and equilibrium unfolding of DeNovoTIMs. (A) Far-UV CD spectra. (B) Near-UV CD spectra. (C) Intrinsic Fluorescence (IF) spectra $\left(\lambda_{\text {exc }}=295 \mathrm{~nm}\right)$. (D) Thermal unfolding followed by $\mathrm{CD}_{222 \mathrm{~nm}}$ (scan rate: $1.5 \mathrm{~K} \mathrm{~min}^{-1}$ ). (E) Differential Scanning Calorimetry (DSC) endotherms (scan rate: $1.5 \mathrm{~K} \mathrm{~min}^{-1}$; for easy comparison, the physical and chemical baselines have been subtracted). F) DSC endotherms of DeNovoTIM14 in the presence of increasing concentrations of urea $\left(2.0\right.$ to $6.0 \mathrm{M}$ ) from bottom to top (scan rate: $1.5 \mathrm{~K} \mathrm{~min}^{-1}$ ). For clarity, in panels $E$ and $F$ only a small part of the pre- and post-transition baselines are shown. (G) Chemical unfolding using urea and followed by CD (notice that DeNovoTIM14 does not unfold with urea). (H) Chemical unfolding induced by guanidinium hydrochloride for DeNovoTIM14 (squares: CD, circles: IF).

reported in the supporting information (Figure S2 and Tables S1-S4).

\section{Folding thermodynamics of DeNovoTIMs}

All variants presented the characteristic far-UV $C D$ spectra observed for $\alpha / \beta$ proteins (Figure 2 (A) and Figure S4). The near-UV CD and intrinsic fluorescence (IF) spectra showed that the aromatic residues are buried from solvent and structured in the folded state (Figure 2(B)-(C) and Figures S5-S6; see supporting text for details). All DeNovoTIMs are monomeric and compact as revealed by analytical size exclusion chromatography (Table S5). ${ }^{33}$

Thermal unfolding was then studied by $C D$ and DSC (Figure 2(D)-(E)). All DeNovoTIMs showed cooperative transitions with a remarkably broad range of $T_{m}$ values, from $47{ }^{\circ} \mathrm{C}$ (DeNovoTIM0) to $109^{\circ} \mathrm{C}$ (DeNovoTIM12) (Table 1); indeed at $90^{\circ} \mathrm{C}$ many of the proteins still showed secondary and tertiary structure (Figure S4(B) and Figure S5(B)). All DeNovoTIMs, except 13 and 14, showed thermal unfolding reversibility (Figure S7) and were well fitted to the two-state model $(\mathrm{N} \leftrightharpoons \mathrm{U})$ (Figure S8 and Table 1). This is remarkable because the temperature-induced unfolding of natural proteins of this size, particularly TIM barrels, is usually not reversible. ${ }^{22,30}$ DeNovoTIM14 showed two endotherms, suggesting the presence of an unfolding intermediate (Figure $S 8(I)$ ). For DeNovoTIM 13 and 14, endotherms were wellfitted to an irreversible two-state mechanism $(\mathrm{N} \rightarrow \mathrm{F})$ giving activation energies $\left(E_{a c t}\right)$ of 120 and $37 \mathrm{kcal} \mathrm{mol}^{-1}$ (Table 1), respectively, resulting in very different kinetic stabilities (Figures S9-S10 and supporting text).

For DeNovoTIMs with a reversible thermal unfolding, the observed unfolding $\Delta H$ and $\Delta C_{P}$ also vary greatly (Table 1$)$. For some 
Table 1 Thermodynamic properties of DeNovoTIMs.

\begin{tabular}{|c|c|c|c|c|c|c|c|c|c|}
\hline \multirow{2}{*}{$\begin{array}{l}\text { de novo TIM } \\
\text { barrel }\end{array}$} & \multicolumn{6}{|c|}{ Thermal unfolding (by DSC) ${ }^{a}$} & \multicolumn{3}{|c|}{ Chemical unfolding (by CD and IF) } \\
\hline & $T_{m}\left({ }^{\circ} \mathrm{C}\right)$ & $\begin{array}{l}\Delta H(\mathrm{kcal} \\
\left.\mathrm{mol}^{-1}\right)\end{array}$ & $\begin{array}{l}\Delta H_{85^{\circ} \mathrm{C}}(\mathrm{kcal} \\
\left.\mathrm{mol}^{-1}\right)^{\mathrm{b}}\end{array}$ & $\begin{array}{l}\Delta C_{P}\left(\mathrm{kcal}^{-1}\right. \\
\left.\mathrm{mol}^{-1} \mathrm{~K}^{-1}\right)\end{array}$ & $\Delta \boldsymbol{H}_{\mathrm{vH}} / \Delta \boldsymbol{H}$ & $\begin{array}{l}\text { Global thermodynamic } \\
\text { stability }\left(\mathrm{kcal} \mathrm{K} \mathrm{mol}^{-1}\right)^{\mathrm{c}}\end{array}$ & $\begin{array}{l}\Delta G_{25}{ }^{\circ} \mathrm{C}(\mathrm{kcal} \\
\left.\mathrm{mol}^{-1}\right)\end{array}$ & $\begin{array}{l}m\left(\mathrm{kcal} \mathrm{mol}^{-1}\right. \\
\left.\mathrm{M}^{-1}\right)\end{array}$ & $\begin{array}{l}D_{[1 / 2]} \\
(M)\end{array}$ \\
\hline sTIM11 & $80.0 \pm 0.2$ & $93 \pm 1$ & $104 \pm 2$ & $2.19 \pm 0.09$ & $0.99 \pm 0.05$ & 280 & $4.8 \pm 0.3$ & $1.34 \pm 0.06$ & 3.1 \\
\hline sTIM11noCys & $65.6 \pm 0.1$ & $82 \pm 1$ & $128 \pm 2$ & $2.36 \pm 0.08$ & $0.99 \pm 0.03$ & 176 & $3.2 \pm 0.2$ & $2.03 \pm 0.10$ & 1.9 \\
\hline DeNovoTIMO & $47.0 \pm 0.2$ & $25 \pm 1$ & $41 \pm 3$ & $0.44 \pm 0.06$ & $1.09 \pm 0.08$ & 61 & $1.5 \pm 0.1$ & $0.76 \pm 0.02$ & 2.1 \\
\hline DeNovoTIM1 & $71.0 \pm 0.4$ & $48 \pm 1$ & $58 \pm 2$ & $0.69 \pm 0.05$ & $0.98 \pm 0.05$ & 226 & $3.8 \pm 0.1$ & $1.87 \pm 0.10$ & 2.0 \\
\hline DeNovoTIM6 & $92.3 \pm 0.1$ & $125 \pm 2$ & $108 \pm 1$ & $2.38 \pm 0.06$ & $1.03 \pm 0.02$ & 542 & $7.9 \pm 0.2$ & $1.51 \pm 0.08$ & 5.6 \\
\hline DeNovoTIM8 & $77.3 \pm 0.3$ & $52 \pm 1$ & $61 \pm 1$ & $1.19 \pm 0.07$ & $0.95 \pm 0.09$ & 165 & $2.5 \pm 0.2$ & $0.85 \pm 0.09$ & 2.9 \\
\hline DeNovoTIM11 & $103.5 \pm 0.2$ & $62 \pm 2$ & $49 \pm 1$ & $0.67 \pm 0.14$ & $1.02 \pm 0.09$ & 523 & $8.4 \pm 0.4$ & $1.75 \pm 0.08$ & 6.0 \\
\hline DeNovoTIM12 & $108.8 \pm 0.3$ & $79 \pm 2$ & $62 \pm 1$ & $0.72 \pm 0.08$ & $1.01 \pm 0.08$ & 792 & $10.9 \pm 0.2$ & $1.77 \pm 0.03$ & 6.2 \\
\hline DeNovoTIM13 & $92.8 \pm 0.4$ & $47 \pm 5$ & \multirow{4}{*}{\multicolumn{2}{|c|}{$\begin{array}{l}\text { ND } E_{\text {act: }}: 120 \pm 3 \mathrm{kcal} \mathrm{mol}^{-1} \mathbf{d} \\
\text { ND } E_{\text {act }} \cdot 37 \pm 1 \mathrm{kcal} \mathrm{mol}^{-1} \mathrm{~d}\end{array}$}} & & ND & $9.5 \pm 0.2$ & $1.54 \pm 0.03$ & 6.6 \\
\hline DeNovoTIM14 & $91.5 \pm 0.1$ & $5.4 \pm 0.2$ & & & & ND & Tot: $23.6 \pm 2.0$ & $\mathrm{~N}-\mathrm{I}: 4.3 \pm 0.9$ & $\mathrm{~N}-\mathrm{I}: 3.2$ \\
\hline & $105.3 \pm 0.1$ & $8.6 \pm 0.4$ & & & & & $\mathrm{~N}-\mathrm{I}: 12.7 \pm 1.9$ & I-U: $2.5 \pm 0.1$ & I-U: 4.4 \\
\hline & & & & & & & I-U:10.9 \pm 0.6 & & \\
\hline
\end{tabular}

ND: Not determined due to irreversibility in the thermal unfolding. Instead, activation energy $\left(E_{\text {act }}\right)$ was calculated from an irreversible two-state mechanism.

${ }^{a}$ The thermodynamic parameters reported are the average of ten experiments carried out at different protein concentrations $\left(0.25-2.5 \mathrm{mg} \mathrm{mL}{ }^{-1} ;\right.$ Figure $\mathrm{S} 8$ and Figure $\left.\mathrm{S} 11\right), \pm$ indicate the standard deviation calculated from these 10 experiments.

${ }^{\mathrm{b}} \Delta H$ at $85^{\circ} \mathrm{C}\left(\Delta H_{85^{\circ} \mathrm{C}}\right)$ was calculated using the experimental $\Delta H$ and $\Delta C_{P}$ values as indicated in material and methods.

${ }^{\mathrm{C}}$ The global thermodynamic stability was calculated from the area of the stability curve evaluated between $0{ }^{\circ} \mathrm{C}$ and $T_{m}$ (Figure $3(\mathrm{~B})$ ).

${ }^{\mathrm{d}} E_{\text {act }}$ value and \pm are the average and standard deviation, respectively, from three different calculation methods (Figure S9 and Figure S10).

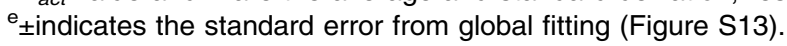


DeNovoTIMs these values are smaller than expected for a protein of 184 residues $(\Delta H=128 \pm$ $4 \mathrm{kcal} \mathrm{mol}^{-1}$ and $\Delta C_{P}=2.6 \pm 0.04 \mathrm{kcal} \mathrm{mol}^{-1} \mathrm{~K}^{-1}$, according to parametric equations reported in, ${ }^{34}$ while the $\Delta H$ values observed for the first and second design rounds $\left(0.24\right.$ to $0.64 \mathrm{kcal} \mathrm{mol}^{-1}$ residue $^{-1}$ ) are similar to those reported for natural monomeric TIM barrels $\left(0.25\right.$ to $0.67 \mathrm{kcal}^{\mathrm{mol}^{-1}}$ residue $\left.^{-1}\right)$. Obtained $\Delta C_{P}$ values were independent of protein concentration and showed a small standard deviation (Figure S8, Figure S11, and Table 1). A decrease in $\Delta C_{P}$ has been shown to result from residual structure in the unfolded state. ${ }^{35}$ This is observed in the far-UV CD spectra of those DeNovoTIMs that are unfolded at $90^{\circ} \mathrm{C}$. In addition, the low $\Delta H$ of DeNovoTIM14 increases in the presence of urea (Figure 2(F) and Figure S10). These results suggest that for some DeNovoTIMs, the reason for the low $\Delta H$ and $\Delta C_{P}$ is likely the high content of residual structure in the unfolded state (supporting text).

Stability at $25{ }^{\circ} \mathrm{C}$ was studied by chemical unfolding with urea or $\mathrm{GdnHCl}$. Except for DeNovoTIM14, all designs were completely unfolded in $9.0 \mathrm{M}$ urea (Figures S4-S6). For all designs, except for DeNovoTIM14, CD and IF curves were monophasic, cooperative, coincident, and globally-fitted well to a two-state $\mathrm{N} \leftrightharpoons \mathrm{U}$ model, indicating the absence of populated intermediates (Figure 2(G) and Figures S12S13). DeNovoTIM14 showed no changes in CD or IF signal up to $9.0 \mathrm{M}$ of urea, even after incubation for 5 days (Figures S4-S6). In contrast, in the presence of GdnHCl DeNovoTIM14 showed a three-state unfolding process with a populated intermediate: $\mathrm{N} \leftrightharpoons \mathrm{I} \leftrightharpoons \mathrm{U}$ (Figure $2(\mathrm{H})$ and Figure S13).

All selected first- and second-round designs had a $\Delta G_{25}{ }^{\circ} \mathrm{C}$ higher than DeNovoTIM0, whereas the triple-design, DeNovoTIM14, showed a pronounced increase in stability $\left(\Delta G_{\text {Tot }}=23.6 \mathrm{kcal}\right.$ $\mathrm{mol}^{-1}$; Table 1). The stability change related to the loss of the native state in DeNovoTIM14 $\left(\Delta G_{N}\right.$ -

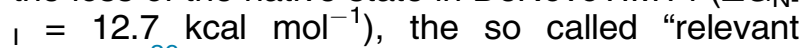
stability", ${ }^{36}$ is higher than the $\Delta G$ of the secondround designs; whereas the stability of the intermediate, also referred as the "residual stability", is similar $\left(\Delta G_{I-U}=10.9 \mathrm{kcal} \mathrm{mol}^{-1}\right)$. For all DeNovoTIMs, the $m$ value, a parameter proportional to the surface area exposed to the solvent upon unfolding $(\triangle \mathrm{ASA}){ }^{37}$ is similar to those observed for natural proteins with the same size, except for DeNovoTIM 0 and 8 where $m$ decreases significantly (Table 1). Low $m$ values have been related to low packing in the native state, residual structure in the unfolded state, or the presence of folding intermediates. ${ }^{38}$ Residual structure in the unfolded state is not clearly observed in the CD spectra of DeNovoTIMs at 9.0 M urea (Figures S4-S5), therefore, other techniques and kinetic studies should help to detect the persistence of native-like structure in the unfolded state and/or the presence of intermediates. ${ }^{38,39}$

The modular approach used in this work improved both $\Delta G$ and $T_{m}$ substantially and hence produced significantly more stable proteins. In this context, it is worth mentioning that over the years the combination of stabilizing mutations has been considered an effective strategy to enhance the stability of small proteins. ${ }^{36,40-43}$ Previous work on small globular proteins with optimized hydrophobic cores and interactions on the surface exhibited increased thermal stability by up to 30 degrees. 9,11,15 Extending these strategies from point mutants to regions appears to be useful for bigger folds such as the TIM barrel. In what follows, using the thermal and chemical unfolding data described above, the thermodynamic properties underlying the stability of DeNovoTIMs are analyzed.

\section{Global thermodynamic stability and non- additive effects of DeNovoTIMs}

As observed in natural proteins, the $m$ values obtained from the chemical unfolding of STIM11, sTIM11noCys, DeNovoTIM 0, 6, and 8 correlate with their $\Delta C_{P}$ values (Figure $3(\mathrm{~A})$ ), likely because both depend on the $\triangle A S A$ upon unfolding. In contrast, $\Delta C_{P}$ values obtained for DeNovoTIM 1, 11, and 12 are much lower than expected (Figure 3(A)). According to the Rosetta models and the native state structures (see below), these differences are not exclusively due to properties of the native state since the calculated $\triangle \mathrm{ASA}$ is close to the expected value for the size of DeNovoTIMs $\left(17135 \mathrm{~A}^{2}\right){ }^{37}$ This suggests that the unfolded state reached at high temperatures is more structured than the one obtained by chemical unfolding.

Thermal unfolding reversibility allowed the assessment of DeNovoTIM stability curves (Figure $3(\mathrm{~B})$ ). The $\Delta G_{25}{ }^{\circ} \mathrm{C}$ values are in excellent agreement with those obtained from chemical unfolding experiments. According to the GibbsHelmholtz equation, conformational stability is modulated by changes in $T_{m}, \Delta H$, and $\Delta C_{P}$. For natural TIM barrels, it has been observed that changes in the stability curve are influenced mainly by modifying one or two of those parameters. ${ }^{30,31}$ In contrast, the DeNovoTIMs differ in all three parameters. Increasing $\Delta H$ is the most commonly found mechanism for stabilization of thermophilic proteins ${ }^{5}$ and is also the most often exploited mechanism for engineering protein stability. ${ }^{7,40}$ In DeNovoTIMs, enthalpy-driven stabilization is found in all proteins but is especially important in DeNovoTIM6 (Figure 3(B)). $\Delta C_{P}$ determines the magnitude of the curvature of the stability curve so that changes in this parameter trigger a more or less flattened curve. A decrease in $\Delta C_{P}$ has been postulated as a mechanism for thermostabilization. ${ }^{35,44}$ 

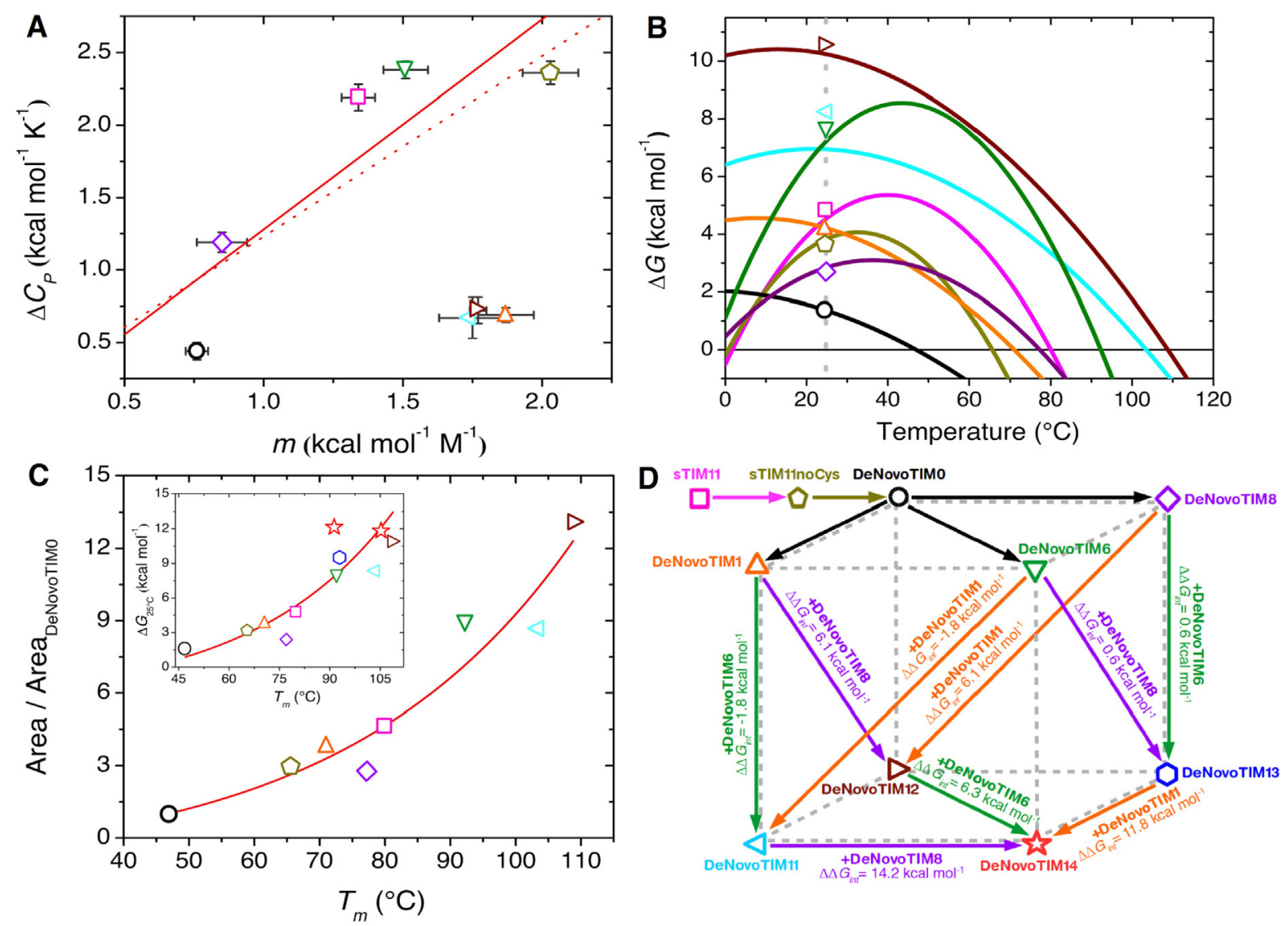

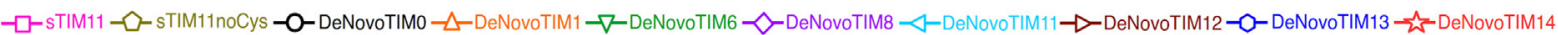

Figure 3. Stability and energetic coupling in DeNovoTIMs. (A) Correlation between two parameters which are proportional to the exposed surface area: $m$ value from chemical unfolding and $\Delta C_{P}$ from temperature-induced unfolding (solid line: linear regression excluding DeNovoTIM1, DeNovoTIM11, and DeNovoTIM12 data; R²: 0.76 . Dotted line: correlation reported by 37). (B) Stability curves calculated from DSC data (lines) using the GibbsHelmholtz equation (open symbols show $\Delta G$ values determined by chemical unfolding at $25^{\circ} \mathrm{C}$. Grey dashed line indicates $25^{\circ} \mathrm{C}$ ). (C) Correlation between the relative global thermodynamic stability (Area/Area DeNovoTIMo) and thermostability $\left(T_{m}\right)\left(\mathrm{R}^{2}: 0.93\right)$. Inset: correlation between $\Delta G$ at $25^{\circ} \mathrm{C}$ determined by chemical unfolding and $T_{m}\left(\mathrm{R}^{2}\right.$ : 0.87). For DeNovoTIM14, where two transitions were found, it was assumed that the one observed at lower [GdnHCl] corresponds to the lower $T_{m}$. (D) Thermodynamic cube showing the coupling energy $\left(\Delta \Delta G_{\text {int }}\right)$ between different regions of DeNovoTIMs. $\Delta \Delta G_{i n t}$ values were calculated from the double-mutant cycles shown in Figure $S 14$. $\Delta \Delta G_{i n t}$ values between single-region mutants are depicted as colored arrows from the top face to the bottom face. $\Delta \Delta G_{i n t}$ values calculated for the addition of a single-region design to a double-region design are shown as colored arrows in the bottom face.

For DeNovoTIMs, the reduction in $\Delta C_{P}$ combined with an increase in $\Delta H$ is the reason for the increase in both $T_{m}$ and $\Delta G_{25}{ }^{\circ} \mathrm{C}$. The results presented here indicate that, as observed for natural proteins, the unfolded ensemble plays an important role in shaping the stability curve and should be considered in protein design.

DeNovoTIMs show a non-linear correlation between $\Delta G_{25}{ }^{\circ} \mathrm{C}$ and $T_{m}$ (inset in Figure $3(\mathrm{C})$ ). A similar trend between $\Delta G$ at the temperature where it is a maximum $\left(\Delta G_{T \max }\right)$ and $T_{m}$ has also been reported for natural and engineered proteins with different sizes and topologies. ${ }^{34,45,46}$ Additionally; the global thermodynamic stability can be con- veniently described by the area (from $0{ }^{\circ} \mathrm{C}$ to $T_{m}$ ) under the stability curve $(A)$. Instead of using a single reference temperature, $A$ integrates the conformational stability in a temperature range. ${ }^{47}$ The relative global stability of DeNovoTIMs $\left(A / A_{\text {DeNovotimo }}\right.$ ) is also correlated with $T_{m}$ (Figure 3 (C)). Notably, for DeNovoTIM 6, 11, and 12, $A / A_{\text {DeNovotimo }}$ is nearly ten-fold higher than the initial design (Figure $3(\mathrm{C})$ and Table 1).

The modular design strategy allowed us to calculate the contribution of each region to global stability, and to evaluate the presence of nonadditive effects between regions of the barrel. Non-additive effects were evaluated as $\Delta \Delta G_{i n t}$ 
through an approach based on thermodynamic double mutant cycles (see material and methods). $\Delta \Delta G_{\text {int }}$ is also referred to as coupling energy, nonadditive effect, interaction energy, and more recently epistatic effect. ${ }^{48}$

Thermodynamic cycles showed that the stabilization is non-additive and depends on the structural context (Figure S14). All the $\Delta \Delta G_{\text {int }}$ values calculated in Figure $\mathrm{S} 14$ are summarized in the single cube shown in Figure 3(D). $\Delta \Delta G_{\text {int }}$ for double designs are much smaller than those involving the triple-region design. The regions that are most energetically coupled in double-region designs $\left(\Delta \Delta G_{\text {int }}=6.1 \mathrm{kcal} \mathrm{mol}^{-1}\right)$ are the internal core (DeNovoTIM1) and the top core (DeNovoTIM8). Coupling increases considerably when a third region is incorporated on top of two already mutated regions $\left(\Delta \Delta G_{\text {int }}>6 \mathrm{kcal} \mathrm{mol}^{-1}\right)$. The largest $\Delta \Delta G_{\text {int }}$ was observed when the DeNovoTIM8 mutations were added to DeNovoTIM11 $\left(\Delta \Delta G_{i n t}=14.2 \mathrm{kcal} \mathrm{mol}^{-1}\right)$ (Figure 3(D) and Figure S14). Clearly, mutations in one place affected other regions of the barrel. The latter indicates that the TIM-barrel fold is suitable for studying modularity and, in general, cooperative effects of proteins. Also, the results presented here suggest that the modular design strategy could be used in the future for the rational stability improvement in other protein topologies.

\section{Structural features of DeNovoTIMs}

The structural properties of DeNovoTIMs were examined by X-ray crystallography (Table S6). High-resolution data were collected for sTIM11noCys and DeNovoTIM13, whereas a lowresolution structure was obtained for DeNovoTIM6. All showed the designed globular compact TIM-barrel topology (Figure 4). Structural comparison with the Rosetta models showed the lowest RMSD located in the second quarter of the barrel. As previously observed in STIM $11,{ }^{29}$ the main structural differences are found in the $\alpha$ helices located at the $\mathrm{N}$ - and $\mathrm{C}$-terminal ends. In agreement, for all the barrel structures, the RMSD among quarters of the barrel is higher in the first and fourth ones (plot in Figure 4(A)). Since the TIM barrel is a closed-repeat protein, contacts between the first and last helices depend on the precise curvature generated by each $\alpha / \beta$ unit, therefore geometrical strain may interfere with the proper closure of the barrel.

A comparison of the sTIM11noCys and STIM11 structures showed that removal of the two cysteines causes some structural changes mainly localized in the first and last quarters; the most significant deviations are observed at the Nterminal region where the first two helices are not well-formed. So even without forming the disulfide bridge, both cysteines in STIM11 increase the stability and promote a proper closure of the barrel (Figure 4(B) and Table 1); nevertheless,
sTIM11noCys maintains the general expected TIM-barrel architecture.

The thermodynamic properties of DeNovoTIM6 are very similar to those expected for a natural protein (Table 1). Unfortunately, due to the low quality of the crystals and therefore the low resolution obtained $(2.9 \AA)$, details such as sidechain conformations are not well resolved in its structure. Nevertheless, it could be verified that the protein is a well folded TIM-barrel (Figure 4 (C)). In DeNovoTIM6, almost all $\alpha / \beta$ loops of the barrel are well defined and correspond to the model. However, for some residues within 5 of the $7 \beta / \alpha$ loops no electron density was observed. In general, the DeNovoTIM6 structure has high B factors which may reflect higher disorder in the protein crystal or increased flexibility, similar to observations in some regions of STIM11, namely the amino- and carboxyl-terminal $\alpha$-helices.

In the DeNovoTIM13 structure, the second and third quarters display only minor differences to the Rosetta model, with the secondary structure elements and side chains superposing very well (Figure 4(D)). In going from sTIM11 to DeNovoTIM13, a $60 \%$ increase in the total area of hydrophobic clusters was found (3765 vs. $6148 \AA^{2}$ ); most of this change comes from a three-fold increase in the area of the major hydrophobic cluster (1116 vs. $4351 \AA^{2}$, Table S7).

As a consequence of the DeNovoTIMs design protocol, polar interactions were replaced by hydrophobic ones, therefore, it is not surprising that the number of $\mathrm{H}$-bonds and salt bridges is lower in DeNovoTIMs than in STIM11 and sTIM11noCys (Table S7). Some of the designs that contained the highest number of polar stabilizing interactions (such as DeNovoTIM 1 and 8) were not the most stable ones, whereas some of the most stable designs (such as DeNovoTIM 6 and 12-14) showed a reduction in this type of interaction (Table S7). In agreement with the design strategy, the stability of DeNovoTIMs increases with the number of hydrophobic interactions. The total area, as well as the number of residues and contacts in hydrophobic clusters, are substantially increased in the best first-round designs along with the more stable second- and third-round designs (Figure S15 and Table S7). This suggests that the strategy of increasing hydrophobic contacts was successful in the stabilization of DeNovoTIMs.

\section{Epistasis on the stability landscape of de novo TIM barrels}

To correlate the most common and informative parameters obtained from both temperature and chemical unfolding, $T_{m}, \Delta H$, and $\Delta G$ were plotted in a two-dimensional bubble plot thereby representing the thermodynamic combinations of the designed proteins (Figure 5). The $T_{m}$ values found in DeNovoTIMs are widely distributed 


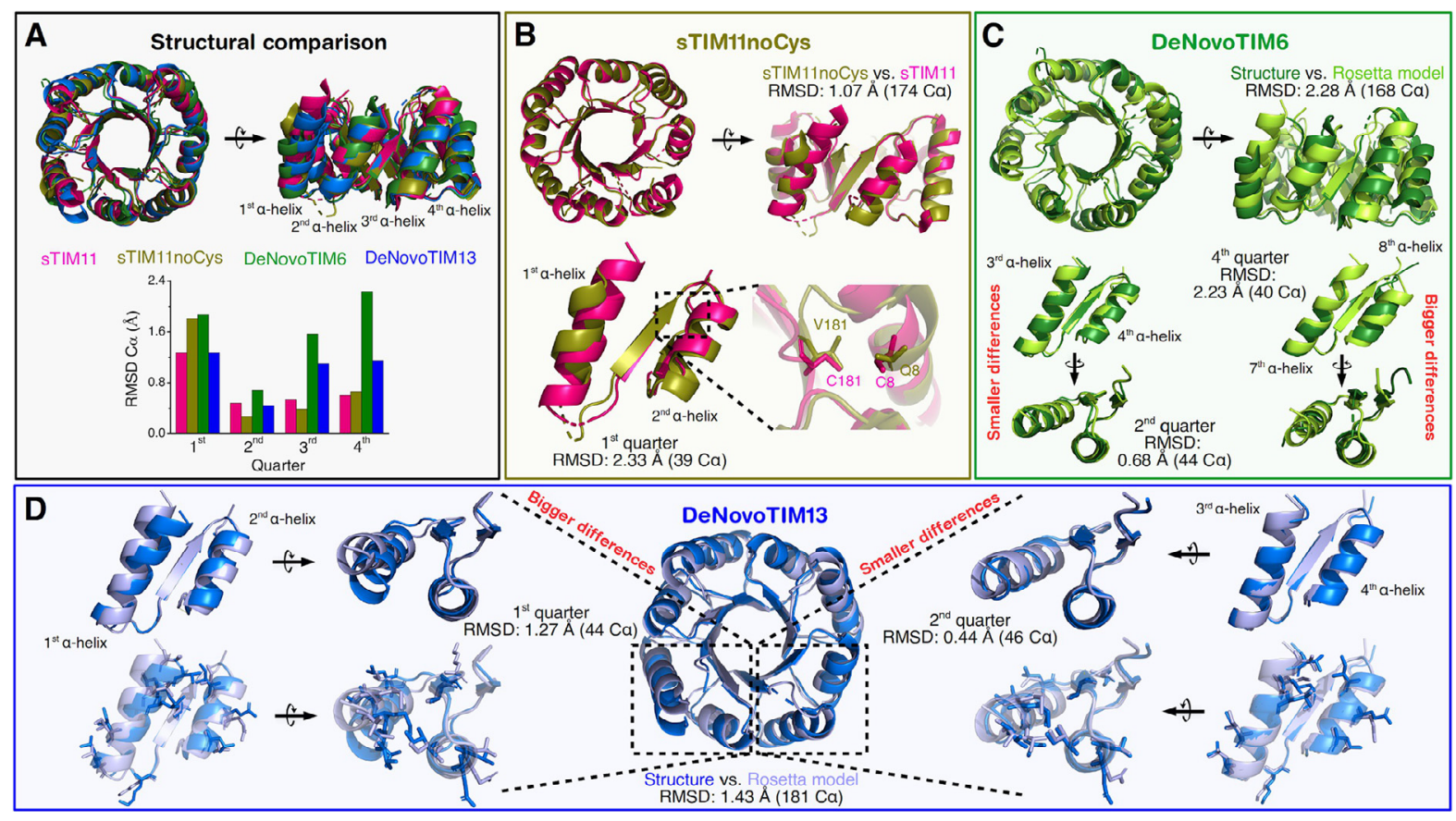

Figure 4. Three-dimensional structures of DeNovoTIMs. (A) Structural alignment of X-ray structures of sTIM11 (PDB ID: 5BVL), sTIM11noCys (PDB ID: 6YQY), DeNovoTIM6 (PDB ID: 6Z2I), and DeNovoTIM13 (PDB ID: 6YQX). The RMSD $\mathrm{C} \alpha$ between the structure and the Rosetta model among the quarters in each protein is shown in the lower part of the panel. (B) Comparison of sTIM11noCys and STIM11 structures (RMSD: $1.07 \AA-174 \mathrm{C} \alpha-$ ). The mutated residues 8 and 181 in STIM11noCys are zoomed in the bottom part. (C) Comparison of the DeNovoTIM6 structure with the Rosetta model (RMSD: $2.28 \AA-168 \mathrm{C} \alpha$-). The quarters with the highest and lowest structural similarity are highlighted (bottom left and bottom right, respectively). (D) Comparison of the DeNovoTIM13 structure with the Rosetta model (RMSD: $1.43 \AA-181 \mathrm{C} \alpha-$ ). The quarters with the highest and lowest structural similarity are highlighted (right and left, respectively). Sidechains of the mutated residues are shown in sticks.

ranging from $47^{\circ} \mathrm{C}$ to $109^{\circ} \mathrm{C}$, corresponding to a $62{ }^{\circ} \mathrm{C}$ increase in thermostability, a range higher than those previously reported for engineered proteins, but in the observed range found in natural proteins (Figure 5). The stability measured from chemical-unfolding experiments $\left(\Delta G_{25^{\circ} \mathrm{C}}\right)$ also shows the variety found in natural TIM barrels and other natural folds (Figure 5(B) and (C)). Natural proteins populate some thermodynamic regions more than others, exploring ample space due to the diversity in size, topology, oligomeric state, function, and evolutionary history. Interestingly, Figure 5 shows that several DeNovoTIMs are located in a region of the plot corresponding to low $\Delta H$ and high $\Delta G_{25^{\circ} \mathrm{C}}$ values, which has not been reported for natural proteins.

Assuming additivity, the expected change in stability calculated for DeNovoTIM14 would be the sum of the individual stabilizations provided by all the single-region designs giving a value of $11.2 \mathrm{kcal} \mathrm{mol}^{-1}$. However, the stability of DeNovoTIM14 is $23.6 \mathrm{kcal} \mathrm{mol}^{-1}$, indicating that more than half of the stabilization comes from positive non-additive effects. Non-additive effects or interaction energies may be referred to as epistasis, a concept traditionally used in genetics to describe the phenotype dependency of a mutation on the genetic state at other sites. ${ }^{48-50}$ Previous studies have explored and analyzed the mechanisms of epistasis within proteins, especially regarding their implications for protein function, evolution, and stability. ${ }^{51-55}$

Rearrangements in the TIM barrel can influence local changes in other parts of the protein, and these epistatic effects are quantified in the $\Delta \Delta G_{i n t}$ values whose magnitude for DeNovoTIMs is considerable. The structural analyses suggest that the epistatic effect observed in DeNovoTIMs is likely related to the extension of the hydrophobic cores, particularly to the increase of the major hydrophobic cluster located in the interface between the inner $\beta$-barrel and the outer $\alpha$-helices (Figure S15 and Table S7). From the first- to the second-round designs, the highest area in hydrophobic clusters was found for DeNovoTIM12, and this corresponds to the highest positive epistatic effect in this round $\left(\Delta \Delta G_{\text {int }}=6.1 \mathrm{kcal} \mathrm{mol}^{-1}\right)$, whereas the decrease of the hydrophobic cluster area in DeNovoTIM11 (compared to DeNovoTIM1 and DeNovoTIM6) correlates with a negative $\Delta \Delta G_{\text {int }}=-1.8 \mathrm{kcal} \mathrm{mol}^{-1}$. From the second- to the third-round designs, the most notable change in hydrophobic area is observed in going from DeNovoTIM11 to 
DeNovoTIM14, resulting in the highest positive epistatic effect $\left(\Delta \Delta G_{\text {int }}=14.2 \mathrm{kcal} \mathrm{mol}^{-1}\right)$. The relevance and magnitude of the epistatic or nonadditive effects found in DeNovoTIMs, as well as those observed in other reports, suggest that modeling such interactions can improve the success in protein design and engineering.

\section{Conclusions}

Design requires a deep understanding of the relationship between sequence, structure, and stability, and therefore, the combination of thermodynamic and structural data is fundamental to achieve this goal. Here, we designed a family of stable TIM barrels and comprehensively explored their thermodynamic and structural properties. The TIM-barrel collection reported in this work exhibits a considerable range in thermostability (more than 60 degrees in $T_{m}$ ) and conformational stability at $25{ }^{\circ} \mathrm{C}$ (more than $22 \mathrm{kcal} \mathrm{mol}^{-1}$ in $\Delta G$ ). These data can now be used to accelerate the development of future custom design protein stability curves which, in turn, will expand the biomedical and biotechnological applications of de novo proteins. For example, by fusion to another de novo protein, one of the stabilized scaffolds reported here (DeNovoTIM13) has been successfully used to create a reaction chamber on the top of the barrel, ${ }^{58}$ confirming the convenience of working with robust and stable TIM barrels in the path towards functional de novo proteins.

In the same way that one explores the sequence space by studying homologous proteins from different organisms, de novo design with a fixed backbone follows a similar strategy generating new sequences within the same topology. It is well known that highly stable proteins can be generated by computational design. However, one of the unexpected findings resulting from the thermodynamic characterization of this family of DeNovoTIMs is that very stable proteins presenting unexplored combinations of thermodynamic parameters can be designed. The stability of DeNovoTIMs is severely influenced by epistatic effects that appear to arise from the design strategy involving an increase in hydrophobic clusters. The design and characterization of stable de novo proteins, such as those described in this work, is an essential step on the route to the next generation of new protein functions charting novel sequence space.

\section{Material and methods}

\section{Enzymes and chemicals}

All reagents were of analytical grade from Merck $\mathrm{KGaA}^{\circledR}$. Genes were ordered from GenScript Biotech. Water was distilled and deionized.

\section{Design protocol}

De novo TIM barrels were designed using the Rosetta software suite v.3.2 ${ }^{59,60}$ (https://www.rosettacommons.org/). All DeNovoTIMs were designed using DeNovoTIMO as a template. The script used for the DeNovoTIM collection follows and executes the steps indicated by the algorithm as indicated in Figure S1. In general, the algorithm first selects the symmetry with which it designs the proteins. Once the two-fold or four-fold symmetry was chosen, it selects the number of residues that mutate (depending on whether it is a quarter or half of the protein). Then, an energy minimization step is performed by simulated annealing considering and evaluating the packing and RMSD. Subsequently, it performs a Monte Carlo (MC) fast layer design to improve the packing of the protein's hydrophobic cores (in one or several cavities selected according to the regions described in Figure 1), minimizes the constraints of the main and side chains, and compares each of them with the starting design. For each step, it verifies the RMSD value between both proteins (in the case of DeNovoTIMs, the design was done with a fixed backbone and a cut-off pointed out $<0.7 \AA$ ). Then, the algorithm filters the results to keep those designs that, with the suggested mutations, were able to increase the packing and preserve the reference topology (ScoreRes: $\leq$ -1.9 , Talaris: $\leq-3.5$ BetaNov, Sspred: $\geq 0.85$, Packstat: $\geq 0.65$ ). To evaluate if the suggested protein folds as expected, selected designs were computationally validated by a later step of forward folding to predict the $a b$ initio three-dimensional structure. The selection was done by an energy score, choosing the designs with the lowest energy value and smallest possible RMSD (located at the bottom left when the energy score against RMSD is plotted). In all selected DeNovoTIMs, funnel plots were observed. Finally, the designs were analyzed and the candidates for experimental characterization were selected based on energy criteria, a fewer number of mutations, and physicochemical properties of the suggested mutations.

\section{Cloning, overexpression, and protein purification}

The nucleotide sequence of all DeNovoTIMs was optimized for expression in Escherichia coli. The coding genes were synthesized and cloned into the pET29b(+) vector by GeneScript (New Jersey, USA), except sTIM11noCys, which was cloned into $\mathrm{pET} 21 \mathrm{~b}(+)$. Proteins were overexpressed in E. coli strain BL21(DE3) $\left(\right.$ Invitrogen $\left.{ }^{\circledR}\right)$ in $1 \mathrm{~L}$ of Terrific Broth (TB) medium supplemented with $30 \mu \mathrm{g} \mathrm{mL}^{-1}$ kanamycin or $100 \mu \mathrm{g} \mathrm{mL}^{-1}$ ampicillin, inoculated with $5 \mathrm{~mL}$ preculture and incubated at $37^{\circ} \mathrm{C}$ and $200 \mathrm{rpm}$. After an $\mathrm{OD}_{600}$ of $0.6-0.8$ was reached, overexpression was induced by adding $1 \mathrm{mM}$ isopropyl-D-1-thiogalactopyranoside (IPTG); growth was continued for $16 \mathrm{~h}$ at $30^{\circ} \mathrm{C}$. After 

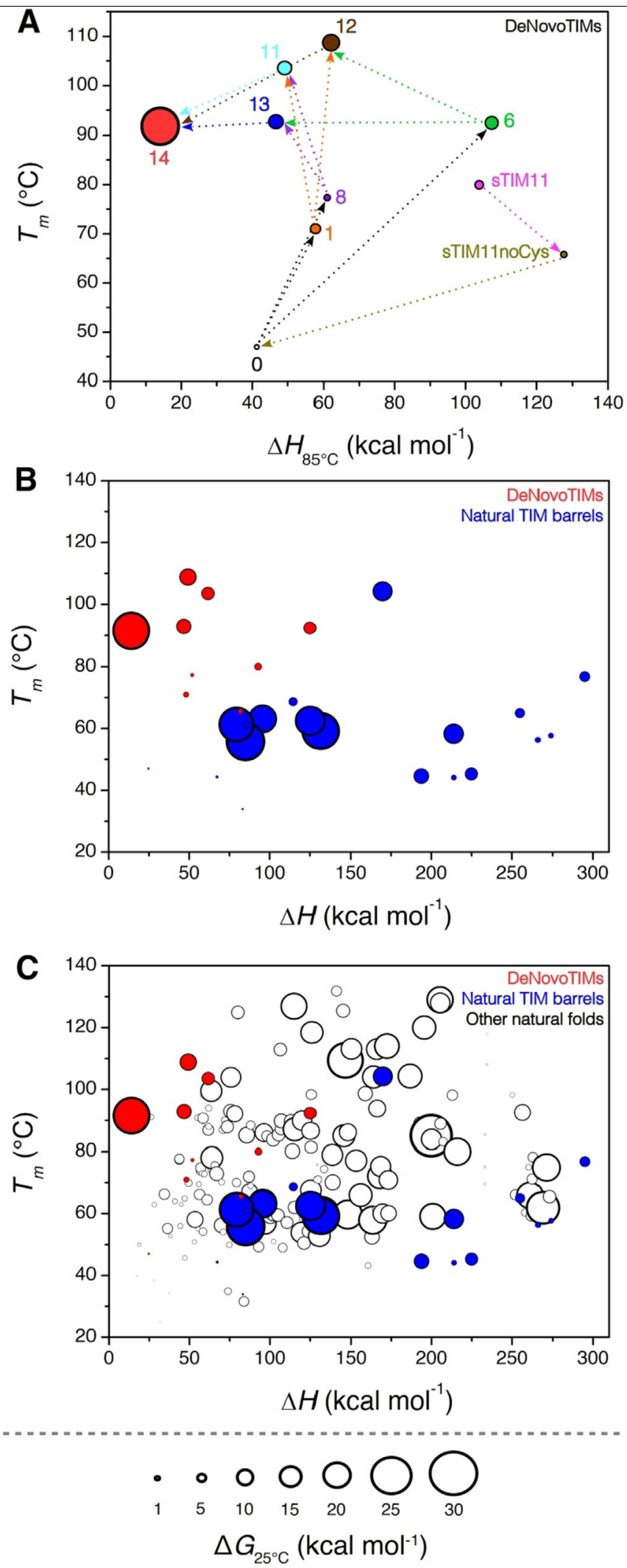

Figure 5. Thermodynamic 2D bubble plots of de novo TIM barrels in comparison with natural proteins. (A) 2D plot of $\Delta H_{85^{\circ} \mathrm{C}}$ versus $T_{m}$ of all DeNovoTIMs. Colored arrows indicate the design flow. (B) 2D plot of $\Delta H$ versus $T_{m}$ of natural TIM barrels (in blue) and DeNovoTIMs (in red). (C) 2D plot as shown in B including data for other natural folded proteins (open circles). The diameter in the bubbles correspond to the $\Delta G_{25^{\circ} \mathrm{C}}$ magnitude. $T_{m}$ and $\Delta H$ data were obtained from thermal unfolding, whereas $\Delta G$ values derive from chemical unfolding. Data for all non-redundant proteins presented here were obtained from ProThermDB database. ${ }^{56,57}$ 
incubation, cells were harvested by centrifugation (Thermo/SLA-3000 ${ }^{\circledR}, 15 \mathrm{~min}, 8000 \mathrm{rpm}, 4^{\circ} \mathrm{C}$ ), pellets resuspended in buffer $\mathrm{A}: 35 \mathrm{mM}$ sodium phosphate, $300 \mathrm{mM} \mathrm{NaCl}$ and $35 \mathrm{mM}$ Imidazole $\mathrm{pH} 8$ (supplemented with $0.2 \mathrm{mM}$ of protease inhibitor Phenylmethylsulfonyl fluoride), lysed by sonication (Cole Parmer Ultrasonic Processor ${ }^{\circledR}$, 10 cycles in $45 \mathrm{~s}$ intervals, $30 \%$ pulse, $4^{\circ} \mathrm{C}$ ), and centrifuged again (Sorvall/SS-34 ${ }^{\circledR}, 40 \mathrm{~min}$, $16000 \mathrm{rpm}, 4^{\circ} \mathrm{C}$ ). In some cases, to increase the efficiency of lysis, the resuspended cells were incubated with lysozyme $\left(250 \mu \mathrm{g} \mathrm{mL}^{-1}\right)$ at $37^{\circ} \mathrm{C}$ for $1 \mathrm{~h}$ before sonication. The purification was performed loading the supernatant onto a HisTrap HP column (5 mL; GE Healthcare Life Sciences ${ }^{\circledR}$ ) coupled to an ÄKTA system (GE Healthcare Life Sciences $\left.{ }^{\circledR}\right)$. The unbound fraction was washed out with 20 column volumes (CV) of buffer A. Bound protein was eluted with a linear gradient of 35-500 mM Imidazole using buffer B: $35 \mathrm{mM}$ sodium phosphate, $300 \mathrm{mM} \mathrm{NaCl}$ and $500 \mathrm{mM}$ Imidazole $\mathrm{pH}$ 8. The pooled fractions were loaded onto a HiLoad 16/600 Superdex 75 preparative grade column (GE Healthcare Life Sciences ${ }^{\circledR}$ ). The proteins were purified using isocratic elution with $1.5 \mathrm{CV}$ of buffer C: $150 \mathrm{mM} \mathrm{NaCl}, 35 \mathrm{mM}$ sodium phosphate $\mathrm{pH}$ 8. The fractions corresponding to the monomeric population were pooled and stored at $4{ }^{\circ} \mathrm{C}$ for use in subsequent experiments. It should be noted that at the end of protein purification, all designs contain a polyhistidine-tag in the carboxyl-terminal region. For DeNovoTIM11 and DeNovoTIM14, the following purification variables were modified to increase the yield: $0.1 \mathrm{mM}$ of IPTG for induction at $\mathrm{OD}_{600}$ of $0.2-0.3, \quad 30{ }^{\circ} \mathrm{C}$ and $6 \mathrm{~h}$ for overexpression, buffer $A$ and $B$ containing $1 \mathrm{M}$ $\mathrm{NaCl}$, and buffer $\mathrm{C}$ with $300 \mathrm{mM} \mathrm{NaCl}$. At each step of the purification process, aliquots were taken to quantify the amount of protein and to calculate the corresponding purification tables. The final yields are indicated in Table S5.

\section{Far- and near-UV circular dichroism}

Circular Dichroism (CD) spectra were collected in buffer D: $10 \mathrm{mM}$ sodium phosphate $\mathrm{pH} 8$ in a Chirascan Spectropolarimeter using a Peltier device to control the temperature (Applied Photophysics ${ }^{\circledR}$ ). For Far-UV spectra, $0.4 \mathrm{mg} \mathrm{mL}^{-1}$ of DeNovoTIM was used for all measurements (1 $\mathrm{nm}$ bandwidth, 185-260 $\mathrm{nm}$ wavelength range, $1 \mathrm{~mm}$ cuvette). For Near-UV spectra, $1 \mathrm{mg} \mathrm{mL}^{-1}$ of DeNovoTIM was used for all measurements (1 $\mathrm{nm}$ bandwidth, 250-350 $\mathrm{nm}$ wavelength range, $10 \mathrm{~mm}$ cuvette). The spectra for thermallyunfolded states were collected at $90^{\circ} \mathrm{C}$. Spectra for chemically-unfolded states were collected at $9 \mathrm{M}$ urea for all DeNovoTIMs, except for DeNovoTIM14, which was collected at $7 \mathrm{M}$ $\mathrm{GdnHCl}$. Raw data were converted to mean residue molar ellipticity $([\theta])$ using: $[\theta]=\theta /\left(\mid C_{C} N_{r}\right)$, where $\theta$ is ellipticity collected in millidegrees, $I$ is the cell path length in $\mathrm{mm}, \mathrm{C}$ is the DeNovoTIM molar concentration, and $\mathrm{N}_{r}$ the number of residues per protein. Far-UV spectra were deconvoluted with CDNN. ${ }^{61}$

\section{Intrinsic fluorescence}

Intrinsic Fluorescence (IF) spectra were collected on a PC1 ISS Spectrofluorometer (Champaign ILUSA $^{\circledR}$ ) equipped with a Peltier device controlling the temperature. In all measurements, protein concentration was $0.4 \mathrm{mg} \mathrm{mL}^{-1}$ in buffer $\mathrm{D}$ : $10 \mathrm{mM}$ sodium phosphate $\mathrm{pH} 8(1 \mathrm{~nm}$ bandwidth slits, $295 \mathrm{~nm}$ excitation wavelength, 310-450 nm emission wavelength range). Spectra for chemically-unfolded states were collected at $9 \mathrm{M}$ urea for all DeNovoTIMs, except for DeNovoTIM14, which was collected at $7 \mathrm{M}$ $\mathrm{GdnHCl}$. Fluorescence spectral center of mass (SCM) was calculated from intensity data $\left(I_{\lambda}\right)$ obtained at different wavelengths $(\lambda)$ : $\mathrm{SCM}=$ $\sum \lambda \mathrm{I}_{\lambda} / \sum \mathrm{I}_{\lambda}$

\section{Three-dimensional structure determination}

DeNovoTIMs were concentrated with Amicon Ultra centrifugal filter units (Millipore ${ }^{\circledR}$ ) and dialyzed in buffer C: $10 \mathrm{mM}$ sodium phosphate $\mathrm{pH}$ $8,150 \mathrm{mM} \mathrm{NaCl}$. Sitting-drop vapor-diffusion method, and JCSG Core I-IV, JCSG +, Classics III, PACT, PEGs I-II, and $\mathrm{AmSO}_{4}$ screening suites $\left(\right.$ Qiagen ${ }^{\circledR}$ ) were used to screen crystallization conditions in 96 well Intelli plates (Art Robbins Instruments $\left.{ }^{\circledR}\right)$ stored at $20{ }^{\circ} \mathrm{C}$ in the hotel-based Rock Imager RI 182 (Formulatrix ${ }^{\circledR}$ ). $0.8 \mu \mathrm{L}$ drops were prepared in a 1:1 ratio with mother liquid using a nanodispensing crystallization robot Phoenix (Art Robbins Instruments ${ }^{\circledR}$ ) and then optimized by multiple crystallization rounds using a sitting-drop vapor-diffusion method. To improve the diffraction quality of DeNovoTIM crystals, different pre- and post-crystallization methods were used: reductive methylation (JBS Methylation Kit, Jena Biosciences ${ }^{\circledR}$ ), seeding, additive screening, controlled dehydration, cryoprotection screening, crystal annealing, and room-temperature diffraction. In total, more than 300 different crystals in various conditions were tested.

Suitable crystals for X-ray diffraction were found in the following conditions: sTIM11noCys: $0.2 \mathrm{M}$ Ammonium Sulfate, $0.1 \mathrm{M}$ Trisodium Citrate $\mathrm{pH}$ 5.6, $25 \% \mathrm{w} / \mathrm{v}$ Polyethylene glycol (PEG) 4000, with a protein concentration of $15 \mathrm{mg} \mathrm{mL}^{-1}$; DeNovoTIM6: $0.095 \mathrm{M}$ Sodium Citrate $\mathrm{pH}$ 5.0, $19 \% \mathrm{v} / \mathrm{v}$ Isopropanol, 25\% w/v PEG 4000, 5\% v/v Glycerol, with a protein concentration of $8.6 \mathrm{mg} \mathrm{mL}^{-1}$; DeNovoTIM13: $0.17 \mathrm{M}$ Sodium Acetate, $0.085 \mathrm{M}$ Tris $\mathrm{pH} 8.5,25.5 \% \mathrm{w} / \mathrm{v}$ PEG $4000,15 \% \mathrm{v} / \mathrm{v}$ Glycerol, with a protein concentration of $10 \mathrm{mg} \mathrm{mL}^{-1}$. 
For sTIM11noCys and DeNovoTIM13, diffraction data were collected at $100 \mathrm{~K}$ at the Swiss Light Source at the Paul Scherrer Institute in Villigen (Switzerland) (X10SA-PXII beamline for sTIM11noCys and X06DA-PXIII beamline for DeNovoTIM13) using a wavelength of $1 \AA$ and a PILATUS 6M detector for sTIM11noCys and a PILATUS 2M-F detector for DeNovoTIM13. ${ }^{62,63}$ For DeNovoTIM6, diffraction data were collected at $100 \mathrm{~K}$ at the Berlin Electron Storage Ring Society for Synchrotron Radiation beamline 14.2 (BESSY BL14.2) operated by Helmholtz-Zentrum Berlin using a wavelength of $0.91 \AA$ and a PILATUS3S $2 \mathrm{M}$ detector. ${ }^{64}$

Diffraction data were processed with the X-ray Detector Software (XDS) using XDSAPP v.2.0 $0^{65,66}$ for sTIM11noCys and DeNovoTIM13; and DIALS ${ }^{67}$ for DeNovoTIM6. For data reduction; criteria used to cut off the data were the resolution shell with a meanl/sigma(I) between $1-2$ and the best $\mathrm{CC}_{1 / 2}$ according to redundancy and completeness. The structures were solved by molecular replacement with PHASER in the PHENIX software suite v.1.17 $7^{68}$ using sTIM11 (PDB ID: 5BVL) as a starting model for sTIM11noCys and the own Rosetta model for DeNovoTIM6 and DeNovoTIM13. Refinement was done with phenix.refine. ${ }^{68}$ The model was improved by map inspection and iteratively manual rebuilding performed in COOT v.0.9. ${ }^{69}$ The final coordinates were validated with PDB_REDO, ${ }^{70}$ MolProbity v.4.2, ${ }^{71}$ and the Protein Data Bank validation service $^{72}$; in all servers; the 3D-structure satisfied all quality criteria. The coordinates and structure factors were deposited in the PDB with accession codes: 6YQY (sTIM11noCys), 6Z2I (DeNovoTIM6), and 6YQX (DeNovoTIM13). The figures were created using PyMOL Molecular Graphics System v.4.5.0 (Schrodinger, LLC).

\section{Analytical size exclusion chromatography}

Hydrodynamic measurements were performed on a Superdex 75 10/300 GL analytical column coupled to an ÄKTA System (GE Healthcare Life Sciences $\left.{ }^{\circledR}\right)$. All experiments were performed in buffer C: $10 \mathrm{mM}$ sodium phosphate $\mathrm{pH} 8,150 \mathrm{mM}$ $\mathrm{NaCl}$ at $25^{\circ} \mathrm{C}$ and a protein concentration range from 0.01 to $2.0 \mathrm{mg} \mathrm{mL}^{-1}$. Experimental molecular weight, Stokes-radii, and oligomeric state were calculated from elution volumes and a calibration curve derived from 7 different known proteins.

\section{Thermal unfolding followed by circular dichroism}

Temperature-induced unfolding was monitored by $C D$ at $222 \mathrm{~nm}$ as a function of temperature using $0.4 \mathrm{mg} \mathrm{mL}^{-1}$ in buffer $\mathrm{D}$ : $10 \mathrm{mM}$ sodium phosphate $\mathrm{pH} 8$, a heating rate of 1.0 and $1.5 \mathrm{~K} \mathrm{~min}^{-1}$, and a $1 \mathrm{~mm}$ path-length cell. The changes in the $C D$ signal were normalized to the fraction of unfolded molecules $\left(f_{U}\right)$ by:
$f_{U}=\frac{y_{o b s}-\left(y_{N}+m_{N} T\right)}{\left(y_{U}+m_{U} T\right)-\left(y_{N}+m_{N} T\right)}$

where $\mathrm{y}_{\mathrm{obs}}$ is the experimentally observed $C D$ signal at a given temperature, and $\left(y_{N}+m_{N} T\right)$ and $\left(y_{U}+m_{U} T\right)$ are the linear fitting equations corresponding to the native and unfolded regions, respectively. $T_{m}$ values were estimated from normalized data fitted with a Boltzmann-type function:

$f_{U}=\frac{-1}{\left(1+e^{\frac{T_{-} T_{m}}{a}}\right)}+1$

where $a$ is related to the slope of the transition.

\section{Thermal unfolding followed by differential scanning calorimetry}

Differential Scanning Calorimetry (DSC) scans were carried out in a VP-Capillary DSC system (MicroCal $^{\circledR}$, Malvern Panalytical). Samples were prepared by exhaustive dialysis in buffer $\mathrm{D}$ : $10 \mathrm{mM}$ sodium phosphate $\mathrm{pH} 8$ and then degassed at room temperature. To ascertain proper instrument equilibration, two buffer-buffer scans were performed before each protein-buffer scan (Figure S7). Corresponding buffer-buffer traces were subtracted from each endotherm. For all proteins a reheating scan was performed to determine the reversibility or irreversibility of the process (Figure S7). Reversibility percentage was calculated by comparing the calorimetric $\Delta H$ (area under the curve) recovered in the second scan and that obtained in the first one ( $\Delta H_{\text {secondscan }}$ / $\left.\Delta H_{\text {firstscan }}\right)^{*} 100$. To verify that irreversibility was not the result of a too high final scanning temperature, the first scans were also performed heating near the $T_{m}$. For DeNovoTIMs with a reversible thermal unfolding, protein concentration varied from 0.25 to $2.5 \mathrm{mg} \mathrm{mL}^{-1}$ and scan rates from 1 to $3 \mathrm{~K} \mathrm{~min}^{-1}$, except for DeNovoTIM0 where protein concentration varied from 1 to $5 \mathrm{mg} \mathrm{mL}^{-1}$. For DeNovoTIMs with an irreversible thermal unfolding, protein concentration was $1 \mathrm{mg} \mathrm{mL}^{-1}$ and scan rates from 1 to $3 \mathrm{~K} \mathrm{~min}^{-1}$. For DeNovoTIM14 in native conditions, protein concentration was increased to 2.5 and $4.5 \mathrm{mg} \mathrm{mL} \mathrm{mb}^{-1}$ to accurately determine the transition. For DeNovoTIM14 in the presence of urea, all the scans were done at $1 \mathrm{mg} \mathrm{mL}^{-1}$ from 2.0 to $6.0 \mathrm{M}$ urea with samples incubated for $6 \mathrm{~h}$ at $10{ }^{\circ} \mathrm{C}$. Origin v.9.0 (OriginLab Corporation, Northampton, MA, USA.) with MicroCal software was used for data analysis.

\section{Thermodynamic parameters from reversible DSC transitions}

DSC endotherms were fitted to equilibrium twostate model $(\mathrm{N} \leftrightharpoons \mathrm{U})$ :

$C_{P}(T)=B_{0}+B_{1} T+f(T) \Delta C_{P}+\frac{\Delta H(T)}{R T_{m}^{2}}\left[\frac{1-f(T)}{1-n+\frac{n}{f(T)}}\right]$ 
where $B_{0}$ and $B_{1}$ define the slope and intercept of the low-temperature baseline segment, $n$ is the number of subunits in the native protein ( 1 for all DeNovoTIMs) and $f(T)$ is the protein fraction in the folded monomeric state, producing $\Delta H$ (at $\left.T_{m}\right), \Delta C_{P}$, and $T_{m}$. The thermodynamic parameters reported are the average of ten experiments carried out in the 0.25 to $2.5 \mathrm{mg} \mathrm{mL}^{-1}$ range. The van't Hoff enthalpy $\left(\Delta H_{\mathrm{vH}}\right)$ was evaluated by $^{73}$ :

$\Delta H_{v H}=\frac{4 R T_{m}^{2} C_{P, T_{m}}}{\Delta H}$

where $R$ is the universal gas constant, $T_{m}$ is the temperature at which $C_{P}$ is maximal, $C_{P, T m}$ is the heat capacity value at $T_{m}$, and $\Delta H$ is the total calorimetric enthalpy of the endotherm.

\section{Thermodynamic parameters from irreversible DSC transitions}

Calorimetric transitions were adequately described by the two-state irreversible model $(\mathrm{N} \rightarrow \mathrm{F})$ where $\mathrm{N}$ is the native protein and $\mathrm{F}$ is the final state. $^{74,75}$ The kinetic conversion from $\mathrm{N}$ to $\mathrm{F}$ is described by a first-order rate constant $(k)$ changing with temperature according to the Arrhenius equation:

$k=\exp \left[\frac{-E_{a c t}}{R}\left(\frac{1}{T}-\frac{1}{T^{\prime \prime}}\right)\right]$

where $T^{\prime}$ is the temperature at which the $k=1 \mathrm{~min}^{-1}$ and $E_{a c t}$ is the activation energy between the native and the transition states that describes the unfolding process. The apparent heat capacity is given by:

$C_{P}^{A P P}=\frac{\Delta H E_{a c t}}{R T_{m}^{2}} \exp (x) \exp [-\exp (x)] ; x=\frac{E_{a c t}}{R T_{m}^{2}}\left(T-T_{m}\right)$

where $T$ is the temperature and $\Delta H$ is the unfolding enthalpy. The $E_{a c t}$ was also obtained following these two procedures: from the slope of Arrhenius plots, i.e. In $k$ vs. 1/T; and derived from a data consistency test, evaluating the effect of scanning rate $(v)$ on $T_{m}{ }^{76}$

\section{Chemical-induced unfolding}

All experiments were carried out at a protein concentration of $0.1 \mathrm{mg} \mathrm{mL}^{-1}$ in buffer $\mathrm{D}$ : $10 \mathrm{mM}$ sodium phosphate $\mathrm{pH} 8$ at $25^{\circ} \mathrm{C}$. To determine whether urea induced unfolding was reversible, unfolding and refolding experiments were assayed. For unfolding experiments, native DeNovoTIM was the initial state, whereas for refolding, the starting state was the unfolded DeNovoTIM incubated overnight in $9.0 \mathrm{M}$ urea. Thereafter samples were incubated at different concentrations of urea $(0-9.0 \mathrm{M})$, either increasing or decreasing the initial concentration (for unfolding and refolding experiments, respectively). Intrinsic fluorescence of both, unfolding and refolding samples, was measured at different times to determine the equilibrium time. Unfolding and refolding transitions are coincident and the signal does not change after incubation for $12 \mathrm{~h}$, i.e. chemical unfolding is reversible and at equilibrium under the experimental conditions. Once the equilibrium time was found, unfolding experiments with samples incubated for $12 \mathrm{~h}$ and followed by CD and IF were performed as aforementioned. IF data at fixed emission wavelength and CD data at $222 \mathrm{~nm}$ were both collected over 2 minutes at each urea concentration. The changes in IF and CD were normalized to the fraction of unfolded molecules $\left(f_{U}\right)$ by:

$$
f_{U}=\frac{y_{o b s}-\left(y_{N}+m_{N}[\text { urea }]\right)}{\left(y_{U}+m_{U}[\text { urea }]\right)-\left(y_{N}+m_{N}[\text { urea }]\right)}
$$

where $y_{o b s}$ is the experimentally observed IF and CD signal at a given temperature, and $\left(y_{N}+m_{N}\right.$ [urea] $)$ and $\left(y_{U}+m_{U}\right.$ [urea] $)$ are the linear fitting equations corresponding to the native and unfolded regions, respectively. All two-state transitions were fitted to Santoro and Bolen equation ${ }^{77}$ which assumes a twostate model $(\mathrm{N} \rightleftharpoons \mathrm{D})$ :

$$
f_{U}=\frac{\left(y_{N}+m_{N}[\text { urea }]\right)+\left(y_{U}+m_{U}[\text { urea }]\right) e^{\frac{-\Delta G^{H 2 O} O_{-m[\text { urea }}}{R T}}}{1+e^{\frac{\left.-\Delta G^{H 2 O}-\text { m[urea }\right]}{R T}}}
$$

where $\Delta G^{H 2 O}$ is the unfolding free energy in absence of denaturant, $m$ is $\Delta G /[$ urea], $T$ is the temperature of the experiment $\left(25^{\circ} \mathrm{C}\right)$, and $\left(y_{N}+m_{N}\right.$ [urea]) and $\left(y_{U}+m_{U}\right.$ [urea]) are the linear fitting equations for the pre- and post-transition states. The chemical unfolding transitions for DeNovoTIM14 in GdnHCl were fitted to a three-state model with an intermediate:

$f_{U}=\frac{\left(y_{U}+m_{U}[G d n H C l]\right) K_{1} K_{2}+\left(y_{N}+m_{N}[G d n H C l]\right)+\left(y_{l}+m_{l}[G d n H C l]\right) K_{1}}{1+K_{1}+K_{1} K_{2}}$

where $K_{1}=e^{\frac{-\Delta G_{N t o l}-m_{N t o l}[G d n H C l]}{R T}}, K_{2}=e^{\frac{-\Delta G_{l t o U}-m_{l t o u}[G d n H C l]}{R T}}, \Delta G_{N t o l}$ and $\Delta G_{I t o U}$ is the unfolding free energy from native state to intermediate and from intermediate to unfolded state, $m_{N t o l}$ and $m_{\text {Itou }}$ is $\Delta G /[\mathrm{GdnHCl}]$ of each step, $T$ is the temperature of the experiment $\left(25^{\circ} \mathrm{C}\right)$, and $\left(y_{N}+m_{N}[\mathrm{GdnHCl}]\right), \quad\left(y_{l}+m_{l}[\mathrm{GdnHCl}]\right), \quad$ and $\left(y_{u}+m_{U}[\mathrm{GdnHCl}]\right)$ are the linear fitting equations for native, intermediate, and unfolded states, respectively. Similar $\Delta G$ values were obtained when experimental protein concentration was increased five fold, ruling out the possibility of a bimolecular association/folding step.

\section{Stability curve and global thermodynamic stability}

Global stability curves, $\Delta G(T)$, were calculated using the thermodynamic parameters obtained from DSC experiments and the Gibbs-Helmholtz equation $^{78}$ :

$$
\Delta G(T)=\Delta H\left(1-\frac{T}{T_{m}}\right)-\Delta C_{P}\left(T_{m}-T+T \ln \left(\frac{T}{T_{m}}\right)\right)
$$

The area under the stability curve is a measure of the global stability of the protein. ${ }^{47}$ It was calculated

integrating Eq. (10) from the lowest temperature at 
which the protein is in the liquid state i.e. $0{ }^{\circ} \mathrm{C}$ (273.15 K) to $T_{m}$ :

$$
\begin{aligned}
\text { Area }= & \left(\left(\Delta H-T_{m} \Delta C_{P}\right)\left(T_{m}-T\right)\right) \\
& -\left(\frac{\Delta H}{2 T_{m}}-\frac{\Delta C_{P}}{2}\right)\left(T_{m}^{2}-T^{2}\right)+\left(\frac{\Delta C_{P}}{4} T_{m}^{2}\right) \\
& +\frac{\Delta C_{P}}{2}\left(T^{2} \ln \frac{T}{T_{m}}-\frac{T^{2}}{2}\right)
\end{aligned}
$$

\section{Thermodynamic 2D bubble plots}

The 2D bubble plots were constructed by plotting $T_{m}$ and $\Delta H_{85^{\circ} \mathrm{C}}$ obtained from thermal unfolding experiments, and $\Delta G_{25^{\circ} \mathrm{C}}$ obtained from chemical unfolding data. Since DeNovoTIMs have different $T_{m}$ values, experimental $\Delta H$ from DSC experiments cannot be directly compared. To put the thermodynamic parameters on a similar ground for comparison in Figure $5(\mathrm{~A}), \Delta H$ at $85^{\circ}$ C $\left(\Delta H_{85^{\circ} \mathrm{C}}\right)$, which is the average $T_{m}$ of the DeNovoTIM collection, was calculated using $\Delta H$ and $\Delta C_{P}$ from DSC experiments as follows ${ }^{79}$ :

$\Delta H_{85^{\circ} \mathrm{C}}=\Delta H+\Delta C_{P}\left(85^{\circ} \mathrm{C}-T_{m}\right)$

where $T_{m}, \Delta H$ and $\Delta C_{P}$ are the experimental values obtained from DSC experiments for each protein, and $85{ }^{\circ} \mathrm{C}$ is the reference temperature. $\Delta H_{85^{\circ} \mathrm{C}}$ was not calculated for DeNovoTIM13 and DeNovoTIM14, because their irreversible thermal unfolding hampered the determination of $\Delta C_{P}$. The plots were constructed with Origin v.9.0 (OriginLab Corporation, Northampton, MA, USA.) and the diameter in the bubbles correspond to the $\Delta G_{25^{\circ} \mathrm{C}}$ magnitude.

\section{Thermodynamic double-mutant cycles}

To calculate non-additive effects between different DeNovoTIM barrel regions, an approximation based on double mutant cycles was used. ${ }^{48,80,81}$ The thermodynamic cycles were constructed using the experimental $\Delta G_{25^{\circ} \mathrm{C}}$ values obtained from chemical unfolding experiments and linking single-region/double-region designs and double-region/triple-region designs as indicated in Figure S14.

Each corner of the square represents a different DeNovoTIM where the mutations are located in a specific region of the barrel or in a combination of them. For double-region cycles (upper panel), from the first to the second design round, $\Delta G_{1}$ and $\Delta G_{2}$ are the changes in stability produced when a single region of the barrel was mutated, $\Delta G_{3}$ and $\Delta G_{4}$ are the changes in stability generated when the same mutations are evaluated in the background of another first-round design. In the triple-region cycles (lower panel), from the second to the third design round, $\Delta G_{1}$ and $\Delta G_{3}$ are the changes in stability produced when the mutations of a single region are introduced in the background of DeNovoTIMO or in a double-region design, whereas $\Delta G_{2}$ and $\Delta G_{4}$ are the changes in stability generated when a double-region design was incorporated in the background of DeNovoTIMO or in a single region design, respectively.

Considering that $\Delta G$ is a state property, if two regions of the barrel are energetically independent, their effects will be additive and not coupled. Therefore, stability changes linked to a particular region will result in the same values on parallel sides of the square, i.e., $\Delta G_{1}=\Delta G_{3}$ and $\Delta G_{2}=\Delta G_{4}$. Any difference the values on the parallel sides of the squares indicates a deviation from additivity and measures the coupling energy between different regions of the barrel, given by $\Delta \Delta G_{i n t}=\Delta G_{4}-\Delta G_{2}=\Delta G_{3}-\Delta G_{1}$, where $\Delta \Delta G_{i n t}$ values have been referred as coupling energy, non-additive effects, interaction energies, and more recently epistatic effects. ${ }^{48}$ A positive $\Delta G_{i n t}$ indicates that the introduction of favorable interactions has a higher stabilizing effect when a nearby region is already mutated.

\section{Sequence and structural analysis}

Sequence alignment was performed with MAFFT v.7.450 ${ }^{82}$ using the secondary structure information from the sTIM11 structure (PDB ID: 5BVL). Sequence identity was calculated with the SIAS server (Universidad Complutense de Madrid, 2013). Structural alignments and RMSD calculations were performed using PyMOL Molecular Graphics System v.4.5.0 (Schrodinger, LLC). Cavity volumes were calculated with MOLE v. $2.5^{83}$ using a standard probe radius of $5 \AA$ and an interior threshold of $1.1 \mathrm{~A}$ with a non-directed exploration path. The accessible surface area (ASA) was calculated with VADAR v.1.8. ${ }^{84}$ In these analyses; changes in ASA for the unfolded state were calculated with an extended Gly-X-Gly peptide. Hydrogen bonds, as well as salt bridges, were calculated using HBPLUS v.3.06 $6^{85}$ and ESBRI ${ }^{86}$ with default parameters for distances and angles. A salt bridge was assigned when two atoms of opposite charge were observed within $4 \AA$. Hydrophobic clusters (formed by ILV residues) were calculated following an algorithm previously reported by Sobolev ${ }^{87}$ and available in the ProteinTools toolkit developed by Dr. Noelia Ferruz-Capapey from the Höcker Lab. ${ }^{88}$ The analysis considers ILE; VAL; and LEU residues and then recaptures the coordinates of their neighboring atoms. Then, the buried solvent-accessible hydrophobic surface area is calculated, and the cluster's total area is computed by the sum of the individual residue areas that comprise it.

\section{Accession numbers}

Coordinates and structure files have been deposited to the Protein Data Bank (PDB) with 
accession numbers: 6YQY (sTIM11noCys), 6Z2I (DeNovoTIM6), and 6YQX (DeNovoTIM13).

\section{Data and materials availability}

All data to support the conclusions of this manuscript are included in the main text and supporting information.

\section{CRediT authorship contribution statement}

Sergio Romero-Romero: Conceptualization, Methodology, Validation, Formal analysis, Investigation, Data curation, Writing - original draft, Writing - review \& editing, Visualization, Supervision. Miguel Costas: Conceptualization, Formal analysis, Investigation, Resources, Writing - original draft, Writing - review \& editing, Funding acquisition. Daniel-Adriano Silva Manzano: Software, Investigation, Writing - review \& editing. Sina Kordes: Methodology, Investigation, Writing - review \& editing. Erendira Rojas-Ortega: Methodology, Investigation, Writing - review \& editing. Cinthya Tapia: Methodology, Investigation, Writing - review \& editing. Yasel Guerra: Methodology, Investigation, Writing review \& editing. Sooruban Shanmugaratnam: Methodology, Investigation, Writing - review \& editing. Adela Rodríguez-Romero: Methodology, Investigation, Resources, Writing - review \& editing, Supervision, Funding acquisition. David Baker: Conceptualization, Resources, Writing review \& editing, Supervision, Project administration, Funding acquisition. Birte Höcker: Conceptualization, Resources, Writing - original draft, Writing - review \& editing, Supervision, Project administration, Funding acquisition. D. Alejandro Fernández-Velasco: Conceptualization, Formal analysis, Investigation, Resources, Writing - original draft, Writing - review \& editing, Supervision, Project administration, Funding acquisition.

\section{Acknowledgments}

We acknowledge financial support and allocation of beamtime by PSI and HZB. We thank the beamline staff at the SLS and at BESSY for assistance, and LANEM-IQ-UNAM for the support in crystal characterization. We thank María Isabel Velázquez López, Laura lliana Alvarez Añorve, Alma Jessica Díaz Salazar, and Georgina Espinosa Pérez for their competent technical support, Gregor Wiese for generating and crystallizing sTIM11noCys, Noelia FerruzCapapey for her help in the structural analyses, as well as Po-Ssu Huang for his comments on the manuscript. We kindly thank all the members of the Fernandez-Velasco, Höcker, and Baker Labs for their constructive suggestions to improve the research.

\section{Funding}

This work was supported by scholarships from CONACYT (749489 to C.T., 387653, 291062, 14401, and 27897 to S.R.R), UNAM-DGAPAPAPIIT (IN220516 to S.R.R.), UNAM-DGAPA (postdoctoral fellowship to Y.G.), CONACYT and UNAM-DGAPA (sabbatical stay fellowship to D.A. F.V.), and Foundations Alexander von Humboldt and Bayer Science \& Education (Humboldt-Bayer Research Fellowship for Postdoctoral Researchers to S.R.R.). This research was also financed by grants from CONACYT (221169 to A. R.R., 254514 to D.A.F.V.), UNAM-DGAPA-PAPIIT (IN220519 to M.C., IN208418 to A.R.R., IN219519 and IN220516 to D.A.F.V.), and Programa de Apoyo a la Investigación y el Posgrado FQ-UNAM (5000-9018 to M.C.). B.H. gratefully acknowledges financial support by the European Research Council (ERC Consolidator Grant 647548 'Protein Lego') and by HZB to visit the beamlines at BESSY.

\section{Declaration of Competing Interest}

The authors declare that they have no known competing financial interests or personal relationships that could have appeared to influence the work reported in this paper.

\section{Appendix A. Supplementary material}

This article contains supporting information that includes: Supporting text, Supporting Figures S1 to S15, and Supporting Tables S1 to S7. Supplementary data to this article can be found online at https://doi.org/10.1016/j.jmb.2021. 167153.

Received 29 March 2021; Accepted 6 July 2021; Available online 14 July 2021

Keywords:

de novo protein design; protein folding and stability; stability landscape; non-additive effects;

$(\beta / \alpha)_{8}$-barrel

$\dagger$ Current address: Neoleukin Therapeutics, 98102 Seattle, USA.

Abbreviations:

$A$, area under the stability curve; $T_{m}$, midpoint of thermal unfolding; CD, Circular Dichroism; DSC, Differential Scanning Calorimetry; $E_{\text {act }}$, activation energy; $\mathrm{GdnHCl}$, 
guanidinium chloride; IF, Intrinsic Fluorescence; $\Delta \Delta G_{\text {int }}$, Gibbs free energy of coupling; $\Delta H_{85^{\circ} \mathrm{C}}$, change in enthalpy extrapolated at $85^{\circ} \mathrm{C}$

\section{References}

1. Tokuriki, N., Tawfik, D.S., (2009). Stability effects of mutations and protein evolvability. Curr. Opin. Struct. Biol., 19 (5), 596-604. https://doi.org/10.1016/j. sbi.2009.08.003.

2. Bloom, J.D., Labthavikul, S.T., Otey, C.R., Arnold, F.H., (2006). Protein stability promotes evolvability. Proc. Natl. Acad. Sci. U.S.A., 103 (15), 5869-5874. https://doi.org/ 10.1073/pnas.0510098103.

3. Becktel, W.J., Schellman, J.A., (1987). Protein stability curves. Biopolymers, 26 (11), 1859-1877. https://doi.org/ 10.1002/bip.360261104.

4. Nojima, H., Ikai, A., Oshima, T., Noda, H., (1977). Reversible thermal unfolding of thermostable phosphoglycerate kinase. Thermostability associated with mean zero enthalpy change. J. Mol. Biol., 116 (3), 429442. https://doi.org/10.1016/0022-2836(77)90078-X.

5. Razvi, A., Scholtz, J.M., (2006). Lessons in stability from thermophilic proteins. Protein Sci., 15 (7), 1569-1578. https://doi.org/10.1110/ps.062130306.

6. Huang, P.S., Boyken, S.E., Baker, D., (2016). The coming of age of de novo protein design. Nature, 537 (7620), 320327. https://doi.org/10.1038/nature19946.

7. Islam, M.M., Kobayashi, K., Kidokoro, S.I., Kuroda, Y., (2019). Hydrophobic surface residues can stabilize a protein through improved water-protein interactions. FEBS J., 286 (20), 4122-4134. https://doi.org/10.1111/ febs. 14941.

8. Goldenzweig, A., Fleishman, S.J., (2018). Principles of protein stability and their application in computational design. Annu. Rev. Biochem., 87 (1), 105-129. https:// doi.org/10.1146/annurev-biochem-062917-012102.

9. Kim, D.N., Jacobs, T.M., Kuhlman, B., (2016). Boosting protein stability with the computational design of $\beta$-sheet surfaces. Protein Sci., 25 (3), 702-710. https://doi.org/ 10.1002/pro.2869.

10. Huang, P.S., Oberdorfer, G., Xu, C., Pei, X.Y., Nannenga, B.L., Rogers, J.M., DiMaio, F., Gonen, T., Luisi, B., Baker, D., (2014). High thermodynamic stability of parametrically designed helical bundles. Science, 346 (6208), 481-485. https://doi.org/10.1126/science.1257481.

11. Borgo, B., Havranek, J.J., (2012). Automated selection of stabilizing mutations in designed and natural proteins. Proc. Natl. Acad. Sci. U.S.A., 109 (5), 1494-1499. https:// doi.org/10.1073/pnas.1115172109.

12. Kuhlman, B., Baker, D., (2004). Exploring folding free energy landscapes using computational protein design. Curr. Opin. Struct. Biol., 14 (1), 89-95. https://doi.org/ 10.1016/j.sbi.2004.01.002.

13. Kwok, S.C., Hodges, R.S., (2003). Clustering of large hydrophobes in the hydrophobic core of two-stranded $\alpha$ helical coiled-coils controls protein folding and stability. $J$. Biol. Chem., 278 (37), 35248-35254. https://doi.org/ 10.1074/jbc.M305306200.

14. Selvaraj, S., Gromiha, M.M., (2003). Role of hydrophobic clusters and long-range contact networks in the folding of $(\alpha / \beta) 8$ barrel proteins. Biophys. J., 84 (3), 1919-1925. https://doi.org/10.1016/S0006-3495(03)75000-0.
15. Malakauskas, S.M., Mayo, S.L., (1998). Design, structure and stability of a hyperthermophilic protein variant. Nat. Struct. Biol., 5 (6), 470-475. https://doi.org/10.1038/ nsb0698-470.

16. Dahiyat, B.I., Mayo, S.L., (1997). Probing the role of packing specificity in protein design. Proc. Natl. Acad. Sci. U.S.A., 94 (19), 10172-10177. https://doi.org/10.1073/ pnas.94.19.10172.

17. Colón, W., Elöve, G.A., Wakem, L.P., Sherman, F., Roder, $\mathrm{H}$., (1996). Side chain packing of the $\mathrm{N}$ - and C-terminal helices plays a critical role in the kinetics of cytochrome $\mathrm{C}$ folding. Biochemistry, 35 (17), 5538-5549. https://doi.org/ 10.1021/bi960052u.

18. Fersht, A.R., Serrano, L., (1993). Principles of protein stability derived from protein engineering experiments. Curr. Opin. Struct. Biol., 3 (1), 75-83. https://doi.org/ 10.1016/0959-440X(93)90205-Y.

19. Basak, S., Nobrega, P.R., Tavella, D., Deveau, L.M., Koga, N., Tatsumi-Koga, R., Baker, D., Massi, F., Matthews, C. R., (2019). Networks of electrostatic and hydrophobic interactions modulate the complex folding free energy surface of a designed $\mathrm{B} \alpha$ protein. Proc. Natl. Acad. Sci. U. S.A., 116 (14), 6806-6811. https://doi.org/10.1073/ pnas. 1818744116 .

20. Watters, A.L., Deka, P., Corrent, C., Callender, D., Varani, G., Sosnick, T., Baker, D., (2007). The highly cooperative folding of small naturally occurring proteins is likely the result of natural selection. Cell, 128 (3), 613-624. https:// doi.org/10.1016/j.cell.2006.12.042.

21. Sterner, R., Höcker, B., (2005). Catalytic versatility, stability, and evolution of the (betaalpha)8-barrel enzyme fold. Chem. Rev., 105 (11), 4038-4055. https://doi.org/ 10.1021/cr030191z.

22. Braselmann, E., Chaney, J.L., Clark, P.L., (2013). Folding the proteome. Trends Biochem. Sci., 38 (7), 337-344. https://doi.org/10.1016/j.tibs.2013.05.001.

23. Romero-Romero, S., Kordes, S., Michel, F., Höcker, B., (2021). Evolution, folding, and design of TIM barrels and related proteins. Curr. Opin. Struct. Biol., 68, 94-104. https://doi.org/10.1016/j.sbi.2020.12.007.

24. Nagarajan, D., Deka, G., Rao, M., (2015). Design of symmetric TIM barrel proteins from first principles. BMC Biochem., 16 (1), 1-22. https://doi.org/10.1186/s12858015-0047-4.

25. Figueroa, M., Oliveira, N., Lejeune, A., Kaufmann, K.W., Dorr, B.M., Matagne, A., Martial, J.A., Meiler, J., Van de Weerdt, C., (2013). Octarellin VI: using rosetta to design a putative artificial $(\beta / \alpha) 8$ protein. PLOS ONE, 8, (8) https:// doi.org/10.1371/journal.pone.0071858 e71858.

26. Höcker, B., Lochner, A., Seitz, T., Claren, J., Sterner, R., (2009). High-resolution crystal structure of an artificial $(B \alpha)$ 8-barrel protein designed from identical half-barrels. Biochemistry, 48 (6), 1145-1147. https://doi.org/10.1021/ bi802125b.

27. Höcker, B., Claren, J., Sterner, R., (2004). Mimicking enzyme evolution by generating new (betaalpha)8-barrels from (betaalpha)4-half-barrels. Proc. Natl. Acad. Sci. U.S. A., 101 (47), 16448-16453. https://doi.org/10.1073/ pnas.0405832101.

28. Offredi, F., Dubail, F., Kischel, P., Sarinski, K., Stern, a.S., van de Weerdt, C., Hoch, J.C., Prosperi, C., Francois, J.M., Mayo, S.L., Martial, J.A., (2004). De novo backbone and sequence design of an idealized alpha/ protein: evidence of 
stable tertiary structure. J. Mol. Biol., 325 (1), 163-174. https://doi.org/10.1061/S022-2836(02)01206-8.

29. Huang, P.S., Feldmeier, K., Parmeggiani, F., FernandezVelasco, D.A., Hocker, B., Baker, D., (2016). De novo design of a four-fold symmetric TIM-barrel protein with atomic-level accuracy. Nat. Chem. Biol., 12 (1), 29-34. https://doi.org/10.1038/nchembio.1966.

30. Romero-Romero, S., Costas, M., Rodríguez-Romero, A., Fernández-Velasco, D.A., (2015). Reversibility and two state behaviour in the thermal unfolding of oligomeric TIM barrel proteins. Phys. Chem. Chem. Phys., 17 (32), 2069920714. https://doi.org/10.1039/c5cp01599e.

31. Cipolla, A., Delbrassine, F., Da Lage, J.L., Feller, G., (2012). Temperature adaptations in psychrophilic, mesophilic and thermophilic chloride-dependent alphaamylases. Biochimie, 94 (9), 1943-1950. https://doi.org/ 10.1016/j.biochi.2012.05.013.

32. Rudolph, R., Siebendritt, R., Kiefhaber, T., (1992). Reversible unfolding and refolding behavior of a monomeric aldolase from staphylococcus aureus. Protein Sci., 1 (5), 654-666. https://doi.org/10.1002/ pro.5560010511.

33. Uversky, V.N., (1993). Use of fast protein size-exclusion liquid chromatography to study the unfolding of proteins which denature through the molten globule. Biochemistry, 32 (48), 13288-13298. https://doi.org/10.1021/ bi00211a042.

34. Robertson, A.D., Murphy, K.P., (1997). Protein structure and the energetics of protein stability. Chem. Rev., 97 (5), 1251-1267. https://doi.org/10.1021/cr960383c.

35. Robic, S., Guzman-Casado, M., Sanchez-Ruiz, J.M., Marqusee, S., (2003). Role of residual structure in the unfolded state of a thermophilic protein. Proc. Natl. Acad. Sci. U.S.A., 100 (20), 11345-11349. https://doi.org/ 10.1073/pnas. 1635051100 .

36. Lamazares, E., Clemente, I., Bueno, M., VelázquezCampoy, A., Sancho, J., (2015). Rational stabilization of complex proteins: a divide and combine approach. Sci. Rep., 5 (9129), 1-11. https://doi.org/10.1038/srep09129.

37. Myers, J.K., Nick Pace, C., Martin Scholtz, J., (1995). Denaturant $m$ values and heat capacity changes: relation to changes in accessible surface areas of protein unfolding. Protein Sci., 4 (10), 2138-2148. https://doi.org/10.1002/ pro. 5560041020.

38. Spudich, G., Marqusee, S., (2000). A change in the apparent $m$ value reveals a populated intermediate under equilibrium conditions in Escherichia coli ribonuclease $\mathrm{HI}$. Biochemistry, 39 (38), 11677-11683. https://doi.org/ 10.1021/bi000466u.

39. Shortle, D., Ackerman, M.S., (2001). Persistence of nativelike topology in a denatured protein in $8 \mathrm{M}$ urea. Science, 293 (5529), 487-489. https://doi.org/ 10.1126/science. 1060438 .

40. Haruki, M., Tanaka, M., Motegi, T., Tadokoro, T., Koga, Y., Takano, K., Kanaya, S., (2007). Structural and thermodynamic analyses of Escherichia Coli RNase $\mathrm{HI}$ variant with quintuple thermostabilizing mutations. FEBS J., 274 (22), 5815-5825. https://doi.org/10.1111/j.17424658.2007.06104.x.

41. Serrano, L., Day, A.G., Fersht, A.R., (1993). Step-wise mutation of barnase to binase. A procedure for engineering increased stability of proteins and an experimental analysis of the evolution of protein stability. J. Mol. Biol., 233, 305312. https://doi.org/10.1006/jmbi.1993.1508.
42. Stearman, R.S., Frankel, A.D., Freire, E., Liu, B., Pabo, C. O., (1988). Combining thermostable mutations increases the stability of $\lambda$ repressor. Biochemistry, 27 (19), 75717574. https://doi.org/10.1021/bi00419a059.

43. Matsumura, M., Signor, G., Matthews, B.W., (1989). Substantial increase of protein stability by multiple disulphide bonds. Nature, 342 (6247), 291-293. https:// doi.org/10.1038/342291a0.

44. Hart, K.M., Harms, M.J., Schmidt, B.H., Elya, C., Thornton, J.W., Marqusee, S., (2014). Thermodynamic system drift in protein evolution. PLOS Biol., 12, (11) https://doi.org/ 10.1371/journal.pbio.1001994 e1001994.

45. Howell, S.C., Inampudi, K.K., Bean, D.P., Wilson, C.J., (2014). Understanding thermal adaptation of enzymes through the multistate rational design and stability prediction of 100 adenylate kinases. Structure, 22 (2), 218-229. https://doi.org/10.1016/j.str.2013.10.019.

46. Rees, D.C., Robertson, A.D., (2001). Some thermodynamic implications for the thermostability of proteins. Protein Sci., 10 (6), 1187-1194. https://doi.org/ 10.1110/ps.180101.

47. Alfano, C., Sanfelice, D., Martin, S.R., Pastore, A., Temussi, P.A., (2017). An optimized strategy to measure protein stability highlights differences between cold and hot unfolded states. Nat. Commun., 8 (May), 1-9. https://doi. org/10.1038/ncomms15428.

48. Horovitz, A., Fleisher, R.C., Mondal, T., (2019). Doublemutant cycles: new directions and applications. Curr. Opin. Struct. Biol., 58 (Oct), 10-17. https://doi.org/10.1016/j. sbi.2019.03.025.

49. Miton, C.M., Tokuriki, N., (2016). How mutational epistasis impairs predictability in protein evolution and design. Protein Sci., 25 (7), 1260-1272. https://doi.org/10.1002/ pro.2876.

50. Starr, T.N., Thornton, J.W., (2016). Epistasis in protein evolution. Protein Sci., 25 (7), 1204-1218. https://doi.org/ 10.1002/pro.2897.

51. Risso, V.A., Manssour-Triedo, F., Delgado-Delgado, A., Arco, R., Barroso-delJesus, A., Ingles-Prieto, A., GodoyRuiz, R., Gavira, J.A., Gaucher, E.A., Ibarra-Molero, B., Sanchez-Ruiz, J.M., (2015). Mutational studies on resurrected ancestral proteins reveal conservation of sitespecific amino acid preferences throughout evolutionary history. Mol. Biol. Evol., 32 (2), 440-455. https://doi.org/ 10.1093/molbev/msu312.

52. Ashenberg, O., Gong, L.I., Bloom, J.D., (2013). Mutational Effects on stability are largely conserved during protein evolution. Proc. Natl. Acad. Sci. U.S.A., 110 (52), 2107121076. https://doi.org/10.1073/pnas.1314781111.

53. Natarajan, C., Inoguchi, N., Weber, R.E., Fago, A., Moriyama, H., Storz, J.F., (2013). Epistasis among adaptive mutations in deer mouse hemoglobin. Science, 340 (6138), 1324-1327. https://doi.org/ 10.1126/science.1236862.

54. Lynch, V.J., May, G., Wagner, G.P., (2011). Regulatory evolution through divergence of a phosphoswitch in the transcription factor CEBPB. Nature, 480 (7377), 383-386. https://doi.org/10.1038/nature10595.

55. Ortlund, E.A., Bridgham, J.T., Redinbo, M.R., Thornton, J. W., (2007). Crystal structure of an ancient protein: evolution by conformational epistasis. Science, 317 (5844), 1544-1548. https://doi.org/ 10.1126/science.1142819. 
56. Kumar, M.D.S., (2006). ProTherm and ProNIT: thermodynamic databases for proteins and proteinnucleic acid interactions. Nucleic Acids Res., 34 (90001), D204-D206. https://doi.org/10.1093/nar/gkj103.

57. Nikam, R., Kulandaisamy, A., Harini, K., Sharma, D., Michael Gromiha, M., (2021). ProThermDB: thermodynamic database for proteins and mutants revisited after 15 years. Nucleic Acids Res., 49 (D1), D420-D424. https://doi.org/10.1093/nar/gkaa1035.

58. Caldwell, S.J., Haydon, I.C., Piperidou, N., Huang, P.S., Bick, M.J., Sebastian Sjöström, H., Hilvert, D., Baker, D., Zeymer, C., (2020). Tight and specific lanthanide binding in a de novo TIM barrel with a large internal cavity designed by symmetric domain fusion. Proc. Natl. Acad. Sci. U.S.A., 117 (48), 30362-30369. https://doi.org/10.1073/ pnas. 2008535117.

59. Koga, N., Tatsumi-Koga, R., Liu, G., Xiao, R., Acton, T.B., Montelione, G.T., Baker, D., (2012). Principles for designing ideal protein structures. Nature, 491 (7423), 222-227. https://doi.org/10.1038/nature11600.

60. Rohl, C.A., Strauss, C.E.M., Misura, K.M.S., Baker, D., (2004). Protein structure prediction using rosetta. Methods Enzymol., 383, 66-93. https://doi.org/10.1007/978-0-38792738-1_11.

61. Böhm, G., Muhr, R., Jaenicke, R., (1992). Quantitative analysis of protein far UV circular dichroism spectra by neural networks. Protein Eng. Des. Sel., 5 (3), 191-195. https://doi.org/10.1093/protein/5.3.191.

62. Fuchs, M.R., Pradervand, C., Thominet, V., Schneider, R., Panepucci, E., Grunder, M., Gabadinho, J., Dworkowski, F. S.N., Tomizaki, T., Schneider, J., Mayer, A., Curtin, A., Olieric, V., Frommherz, U., Kotrle, G., Welte, J., Wang, X., Maag, S., Schulze-Briese, C., Wang, M., (2014). D3, the new diffractometer for the macromolecular crystallography beamlines of the swiss light source. J. Synchrotron Radiat., 21 (2), 340-351. https://doi.org/10.1107/ S160057751400006X.

63. Bingel-Erlenmeyer, R., Olieric, V., Grimshaw, J.P.A., Gabadinho, J., Wang, X., Ebner, S.G., Isenegger, A., Schneider, R., Schneider, J., Glettig, W., Pradervand, C., Panepucci, E.H., Tomizaki, T., Wang, M., Schulze-Briese, C., (2011). SLS crystallization platform at beamline X06DA-A fully automated pipeline enabling in situ x-ray diffraction screening. Cryst. Growth Des., 11 (4), 916-923. https://doi.org/10.1021/cg101375j.

64. Helmholtz-Zentrum Berlin für Materialien und Energie. (2016). The MX Beamlines BL14 .1-3 at BESSY II. J. Large-Scale Res. Facil. 47 (2), 1-6. http://dx.doi.org/ $10.17815 /$ jlsrf-2-64

65. Kabsch, W., (2010). XDS. Acta Crystallogr. Sect. D Biol. Crystallogr., 66 (2), 125-132. https://doi.org/10.1107/ S0907444909047337.

66. Sparta, K.M., Krug, M., Heinemann, U., Mueller, U., Weiss, M.S., (2016). XDSAPP2.0. J. Appl. Crystallogr., 49, 10851092. https://doi.org/10.1107/S1600576716004416.

67. Winter, G., Waterman, D.G., Parkhurst, J.M., Brewster, A. S., Gildea, R.J., Gerstel, M., Fuentes-Montero, L., Vollmar, M., Michels-Clark, T., Young, I.D., Sauter, N.K., Evans, G., (2018). DIALS: implementation and evaluation of a new integration package. Acta Crystallogr. Sect. D Struct. Biol., 74, 85-97. https://doi.org/10.1107/S2059798317017235.

68. Adams, P.D., Afonine, P.V., Bunkóczi, G., Chen, V.B., Davis, I.W., Echols, N., Headd, J.J., Hung, L.W., Kapral, G. J., Grosse-Kunstleve, R.W., McCoy, A.J., Moriarty, N.W.,
Oeffner, R., Read, R.J., Richardson, D.C., Richardson, J. S., Terwilliger, T.C., Zwart, P.H., (2010). PHENIX: A comprehensive python-based system for macromolecular structure solution. Acta Crystallogr. Sect. D Biol. Crystallogr., 66 (2), 213-221. https://doi.org/10.1107/ S0907444909052925.

69. Emsley, P., Lohkamp, B., Scott, W.G., Cowtan, K., (2010). Features and development of coot. Acta Crystallogr. Sect. D Biol. Crystallogr., 66 (4), 486-501. https://doi.org/ 10.1107/S0907444910007493.

70. Joosten, R.P., Long, F., Murshudov, G.N., Perrakis, A., (2014). The PDB-REDO server for macromolecular structure model optimization. IUCrJ, 1, 213-220. https:// doi.org/10.1107/S2052252514009324.

71. Chen, V.B., Arendall, W.B., Headd, J.J., Keedy, D.A., Immormino, R.M., Kapral, G.J., Murray, L.W., Richardson, J.S., Richardson, D.C., (2010). MolProbity: All-atom structure validation for macromolecular crystallography. Acta Crystallogr. Sect. D Biol. Crystallogr., 66 (1), 12-21. https://doi.org/10.1107/S0907444909042073.

72. Berman, H.M., Battistuz, T., Bhat, T.N., Bluhm, W.F., Bourne, P.E., Burkhardt, K., Feng, Z., Gilliland, G.L., lype, L., Jain, S., Fagan, P., Marvin, J., Padilla, D., Ravichandran, V., Schneider, B., Thanki, N., Weissig, H., Westbrook, J.D., Zardecki, C., (2002). The protein data bank. Acta Crystallogr. Sect. D Biol. Crystallogr., 58 (6 I), 899-907. https://doi.org/10.1107/S0907444902003451.

73. Freire, E., (1994). Statistical thermodynamic analysis of differential scanning calorimetry data: structural deconvolution of heat capacity function of proteins. Methods Enzymol., 240 (C), 502-530. https://doi.org/ 10.1016/S0076-6879(94)40062-8.

74. Lyubarev, A.E., Kurganov, B.I., (1998). Modeling of irreversible thermal protein denaturation at varying temperature. I. The model involving two consecutive irreversible steps. Biochem., 63 (4), 434-440.

75. Sanchez-Ruiz, J.M., (1992). Theoretical analysis of lumryeyring models in differential scanning calorimetry. Biophys. J., 61 (4), 921-935. https://doi.org/10.1016/S0006-3495 (92)81899-4.

76. Costas, M., Rodríguez-Larrea, D., De Maria, L., Borchert, T.V., Gómez-Puyou, A., Sanchez-Ruiz, J.M., (2009). Between-species variation in the kinetic stability of TIM proteins linked to solvation-barrier free energies. J. Mol. Biol., 385 (3), 924-937. https://doi.org/10.1016/j. jmb.2008.10.056.

77. Santoro, M.M., Bolen, D.W., (1988). Unfolding free energy changes determined by the linear extrapolation method. 1. Unfolding of phenylmethanesulfonyl a-chymotrypsin using different denaturants. Biochemistry, 27 (21), 8063-8068. https://doi.org/10.1021/bi00421a014.

78. Kumar, S., Tsai, C.J., Nussinov, R., (2002). Maximal stabilities of reversible two-state proteins. Biochemistry, 41 (17), 5359-5374. https://doi.org/10.1021/bi012154c.

79. Privalov, P.L., Gill, S.J., (1988). Stability of protein structure and hydrophobic interaction. Adv. Protein Chem., 39 (C), 191-234. https://doi.org/10.1016/S0065-3233(08)60377-0.

80. Horovitz, A., Fersht, A.R., (1990). Strategy for analysing the co-operativity of intramolecular interactions in peptides and proteins. J. Mol. Biol., 214 (3), 613-617. https://doi.org/ 10.1016/0022-2836(90)90275-Q.

81. Carter, P.J., Winter, G., Wilkinson, A.J., Fersht, A.R., (1984). The use of double mutants to detect structural changes in the active site of the tyrosyl-TRNA synthetase 
(Bacillus Stearothermophilus). Cell, 38 (3), 835-840. https://doi.org/10.1016/0092-8674(84)90278-2.

82. Katoh, K., Standley, D.M., (2013). MAFFT multiple sequence alignment software version 7: improvements in performance and usability. Mol. Biol. Evol., 30 (4), 772780. https://doi.org/10.1093/molbev/mst010.

83. Pravda, L., Sehnal, D., Toušek, D., Navrátilová, V., Bazgier, V., Berka, K., Vařeková, R.S., Koča, J., Otyepka, M., (2018). MOLEonline: A web-based tool for analyzing channels, tunnels and pores (2018 update). Nucleic Acids Res., 46 (W1), W368-W373. https://doi.org/ 10.1093/nar/gky309.

84. Willard, L., Ranjan, A., Zhang, H., Monzavi, H., Boyko, R. F., Sykes, B.D., Wishart, D.S., (2003). VADAR: A web server for quantitative evaluation of protein structure quality. Nucleic Acids Res., 31 (13), 3316-3319. https:// doi.org/10.1093/nar/gkg565.

85. McDonald, I.K., Thornton, J.M., (1994). Satisfying hydrogen bonding potential in proteins. J. Mol. Biol., 238 (5), 777-793. https://doi.org/10.1006/jmbi.1994.1334.

86. Costantini, S., Colonna, G., Facchiano, A.M., (2008). ESBRI: A web server for evaluating salt bridges in proteins. Bioinformation, 3 (3), 137-138. https://doi.org/ 10.6026/97320630003137.

87. Sobolev, V., Sorokine, A., Prilusky, J., Abola, E.E., Edelman, M., (1999). Automated analysis of interatomic contacts in proteins. Bioinformatics, 15 (4), 327-332. https://doi.org/10.1093/bioinformatics/15.4.327.

88. Ferruz, N., Schmidt, S., Höcker, B., (2021). ProteinTools: a toolkit to analyze protein structures. Nucleic Acids Res., gkab375 https://doi.org/10.1093/nar/gkab375.

89. Woody, R.W., (1978). Aromatic side-chain contributions to the far ultraviolet circular dichroism of peptides and proteins. Biopolymers, 17 (6), 1451-1467. https://doi.org/ 10.1002/bip.1978.360170606.

90. Hilser, V.J., Gómez, J., Freire, E., (1996). The enthalpy change in protein folding and binding: refinement of parameters for structure-based calculations. Proteins Struct. Funct. Genet., 26 (2), 123-133. https://doi.org/ 10.1002/(SICI)1097-0134(199610)26:2<123::AIDPROT2>3.0.CO;2-H.

91. Makhatadze, G., Privalov, P.L., (1992). Protein interactions with urea and guanidinium chloride: a calorimetric study. $J$. Mol. Biol., 226 (2), 491-505. https://doi.org/10.1016/00222836(92)90963-K. 